\title{
Precios internacionales, tipo de cambio y restricción externa: \\ un análisis de equilibrio general computable.
}

\author{
Dante Nicolás Quaglia \\ Tesis de Maestría \\ Maestría en Economía \\ Universidad Nacional de La Plata
}

Director de Tesis: Martín Cicowiez

2 de diciembre de 2015

Clasificación JEL: C68, E27 


\title{
Precios internacionales, tipo de cambio y restricción externa: un análisis de equilibrio general computable.
}

\author{
Dante Nicolás Quaglia †
}

\section{Resumen}

El presente trabajo analiza el efecto de la caída de los precios internacionales de las commodities sobre la economía argentina. Utilizando la metodología de equilibrio general computable se construyeron dos modelos y se evaluó el rol del tipo de cambio y la restricción externa al utilizar distintos supuestos. El primer modelo tiene las características estándar para países en desarrollo, y el segundo utiliza los principales supuestos estructuralistas. En ambos modelos el shock exógeno tuvo efectos contractivos. En el modelo estándar el ajuste del tipo de cambio real reduce el impacto negativo sobre el PBI, mientras que en el modelo estructuralista la ausencia de ajustes automáticos hace más profunda la caída del producto. Esta última especificación permite cuantificar la necesidad de financiamiento externo y evaluar políticas para administrar la restricción externa.

\begin{abstract}
This paper analyzes the effect of a reduction in international commodity prices on the Argentinian economy. Using the CGE methodology two models were constructed. It was evaluated the role of the exchange rate and the external constraint using different assumptions. The first model has the standard features for developing countries, and the second uses the main structuralist assumptions. In both models, the exogenous shock had contractionary effects. In the standard model the adjustment of the real exchange rate reduces the negative impact on GDP, while in the structuralist model the absence of automatic adjustments deepens the fall in output. The latter specification allows to quantify the need for external financing and the evaluation of policies to manage the external constraint.

Clasificación JEL: C68, E27

Palabras clave: Equilibrio General Computable, Estructura Económica, Tipo de Cambio, Restricción Externa.

\footnotetext{
* Este trabajo constituye la tesis de Maestría en Economía de la Universidad Nacional de La Plata (UNLP), realizada bajo la dirección de Martín Cicowiez. El autor agradece su valioso apoyo y dedicación. Los errores u omisiones son de exclusiva responsabilidad del autor.

${ }^{\dagger}$ Universidad Nacional de La Plata, mail: dnquaglia@hotmail.com.
} 


\section{Índice}

1. Introducción 1

2. Los modelos de equilibrio general computable. 2

2.1. Antecedentes . . . . . . . . . . . . . . . . . . 3

2.2. Los supuestos en los modelos CGE . . . . . . . . . . . . . . . 4

2.3. Los cierres macroeconómicos . . . . . . . . . . . . . . . . . 5

2.4. Los supuestos en los modelos estándar y estructuralista . . . . . . . 7

2.5. Especificaciones del modelo estructuralista . . . . . . . . . . . . . 8

3. La estructura económica argentina 9

3.1. La Matriz de Contabilidad Social . . . . . . . . . . . . . . . . . 9

4. Los modelos estándar y estructuralista 13

4.1. Ecuaciones principales .................. . 13

$\begin{array}{ll}\text { 5. Simulaciones } & 18\end{array}$

5.1. Análisis del comercio internacional . . . . . . . . . . . . . . . . 21

5.2. Reducción de retenciones a las exportaciones . . . . . . . . . . . 25

5.3. El tipo de cambio como herramienta de política . . . . . . . . . 27

$\begin{array}{lr}\text { 6. Conclusiones } & 28\end{array}$

A. ANEXO 1: Los modelos 34

B. ANEXO 2: Especificaciones y elasticidades 44 


\section{Introducción}

La economía argentina se ha caracterizado históricamente por la abundancia en sus recursos naturales. Esta particular dotación de factores derivó en el elevado protagonismo que los productos primarios y sus derivados han tenido en el entramado productivo. A fines del siglo diecinueve, la abundancia de tierras fértiles y el interés de los países centrales en establecer los roles del comercio internacional resultaron en una inserción mundial basada en la exportación de productos agropecuarios e importación de productos manufacturados.

No obstante, la estructura industrial argentina se fue diversificando a través de los años, y a fines de los cuarenta la industria manufacturera alcanzó un papel preponderante en la economía, configurando una estructura productiva compuesta por dos grandes sectores: el agropecuario y el industrial. El primero, altamente competitivo producto de la abundancia de recursos naturales, y el segundo, menos competitivo a nivel mundial, pero fundamental para la inclusión de gran parte de la población al circuito económico (Diamand, 1972). Así fue como la expansión industrial de mediados de siglo tuvo entre sus características distintivas la fuerte dependencia de insumos importados para la producción de bienes industriales (Rapoport, 2008). En consecuencia, el crecimiento económico ha estado siempre condicionado a la obtención de los recursos necesarios para financiar las importaciones de bienes intermedios y de capital, imprescindibles para el funcionamiento de la industria doméstica.

La última década estuvo signada por ésta dinámica, propia de la economía argentina. La recesión iniciada en 1999 terminó en una crisis económica y social sin precedentes, y la Argentina entró al tercer milenio sumergida en profundas dificultades económicas, políticas e institucionales. Luego de la crisis de 2001 y 2002, la economía entró en una elevada senda de crecimiento, que permitió recomponer la estructura productiva luego de varios años de recesión. La rápida reactivación se debió a la combinación de múltiples factores, entre los que se encuentran la elevada capacidad ociosa y alto desempleo en que se hallaba la economía, el limitado pass through tras la devaluación del 2002, que permitió mantener un tipo de cambio real elevado, el aumento de los precios internacionales de las commodities y la mejora en los términos de intercambio, el crecimiento del gasto público, entre los más importantes. Para el año 2008, la propia dinámica de crecimiento reavivó los viejos problemas de la economía argentina. La utilización del tipo de cambio como ancla nominal y el aumento de los precios internos fueron socavando la competitividad cambiaria. A esta situación se le sumó el creciente déficit energético y la baja en los precios de los principales 
productos de exportación registrada en 2014. En este marco, la restricción externa, presente desde los años cincuenta en casi todas las etapas del crecimiento argentino, volvió a ser un serio limitante para el desarrollo (Basualdo, 2006).

La presente investigación explora la sensibilidad de la economía argentina ante cambios en los precios de los principales productos de exportación de Argentina, haciendo foco en los precios de las commodities. Posteriormente se evalúan alternativas de política para hacer frente a estos shocks exógenos. Para analizar cómo afectan estos cambios al conjunto de la economía se construyeron dos modelos de equilibrio general computable: el primero es un modelo estándar para países en desarrollo, y el segundo utiliza los principales supuestos de la escuela estructuralista. De esta manera es posible valorar los diferentes resultados que un mismo shock puede tener en la economía de acuerdo a los supuestos micro y macroeconómicos utilizados. La investigación se estructura del siguiente modo: a continuación se describe la metodología utilizada, el marco teórico, los trabajos previos y antecedentes; en la tercer sección se realiza una breve descripción de la estructura económica argentina para el año 2012 -año base del modelo- y se detalla la matriz de contabilidad social utilizada para su calibración; en la cuarta sección se definen las características centrales de los modelos utilizados; en la sección cinco se presentan los resultados de las simulaciones; y en la sección seis se exponen las conclusiones.

\section{Los modelos de equilibrio general computable.}

Los modelos de equilibrio general computable (Computable General Equilibrium, CGE) consisten en la construcción de sistemas económicos que consideran la interacción de múltiples mercados, en donde están presentes las relaciones entre todos los agentes económicos que forman parte de dicho sistema. Son habitualmente utilizados para evaluar el impacto de diferentes shocks exógenos en el conjunto de la economía, ya que la modelación a través del equilibrio general permite simular los efectos que un cambio produce en todo el sistema. (Robinson, 2006). A diferencia de los modelos de equilibrio parcial, que captan únicamente los efectos que se producen en el mercado bajo estudio, los modelos de equilibrio general permiten evaluar los efectos directos e indirectos que un cambio exógeno produce en la economía.

Existen a grandes rasgos dos corrientes de modelos CGE, los de tipo neoclásico y los estructuralistas, siendo ampliamente más desarrollados aquellos de raíz neoclásica. Debido a las características particulares de los países en vías de desarrollo, los 
modelos CGE neoclásicos incorporan en mayor o menor medida características estructurales de las economías emergentes. Estos modelos estándar son actualmente los más utilizados para los países en desarrollo. En ellos se simulan economías abiertas para países pequeños (De Melo y Robinson, 1989), y aunque mantienen los rasgos centrales de la concepción neoclásica, incorporan aspectos estructurales específicos de las economías bajo estudio.

Por su parte, los modelos estructuralistas se caracterizan por considerar que las causas estructurales de una economía definen su comportamiento. Uno de sus principales exponentes, Lance Taylor (1990), los define de la siguiente manera: “. . [En estos modelos] los recursos naturales, el capital y el trabajo se entrelazan a través de procesos históricos e institucionales. Estos definen los regímenes de propiedad, configurando una heterogénea composición de las clases sociales, y la distribución del ingreso de la economía es fruto de tensiones entre los distintos actores que componen el conjunto económico...". Si bien algunas de estas características también pueden encontrarse en los modelos estándar, una diferencia importante radica en que en general la macrocausalidad fluye desde el lado de la demanda. De esta manera, dado un stock de capital, un aumento en la demanda efectiva puede incrementar el producto y el empleo a la vez que sube la utilización de capital (Taylor, 2004). Los actores económicos tienen poder de mercado, y los empresarios, trabajadores, terratenientes y gobierno interactúan influyendo en los precios y cantidades utilizando su capacidad de negociación. La distribución del ingreso resultante se establece mediante arreglos institucionales, y está en constante tensión debido a los distintos intereses de las clases sociales. En síntesis, los CGE estructuralistas hacen hincapié en el carácter estructural de la economía para definir las relaciones económicas, incluyen poder de mercado por parte de las empresas, desempleo en los factores productivos, y asignan a las instituciones un rol importante en la configuración de la economía.

\subsection{Antecedentes}

Luego del trabajo precursor desarrollado por Johansen (1960), los modelos CGE para los países en desarrollo comenzaron a aplicarse a mediados de los años 70 . Dentro de los modelos estándar, ubicados en la tradición neoclásica-estructuralista, se encuentran los artículos publicados por Dervis et al. (1982), De Melo y Robinson (1989), Robinson (1989), entre otros. Más recientemente, Lofgren et al. (2002) elaboraron el modelo estándar IFPRI, que gracias a su formato ha sido aplicado para 
evaluar los shocks en numerosos países. Por su parte, los modelos estructuralistas tuvieron su desarrollo con trabajos tales como Taylor (1980) para Brasil, y Taylor (1990), donde se recopila una serie de modelos estructuralistas para países en desarrollo. Más recientemente, Taylor (2006) compara los resultados obtenidos al aplicar diferentes supuestos ante un shock de liberalización comercial. Von Arnim (2009) estudia el impacto de la crisis financiera en EEUU, y Von Arnim y Rada (2011) realizan un modelo aplicado para Egipto, donde analizan la relación en el uso de la energía y la productividad laboral.

En Argentina la producción de modelos CGE no se encuentra muy desarrollada, siendo la última década el período más fructífero en trabajos que utilizan esta metodología de análisis. Sin ánimo de agotar la literatura, se pueden encontrar varios artículos que abordan desde la modelación CGE diferentes temáticas. Di Gresia (2009), investiga los cambios en la estructura tributaria, desagregando al sector gobierno en sector nacional y provincial. Chisari et al. (2009) avanza en la formulación de un modelo real financiero. Cicowiez y Mercado (2009) analizan el impacto de la crisis financiera del 2008. Serino (2009) construye un modelo estructuralista para evaluar shocks externos en un contexto de país pequeño con abundancia en recursos naturales. Una de las conclusiones de este trabajo es que el aumento de precios de las commodities impacta negativamente en el producto. Cicowiez (2011) realiza un modelo dinámico recursivo con el objetivo de analizar el impacto, tanto de corto como de largo plazo, de diversos shocks exógenos sobre la economía. Finalmente, Chisari et al. (2014) construye un modelo para evaluar el impacto de la caída en los precios de las commodities para Argentina, Chile, Brasil y Uruguay. En este trabajo el PBI se reduce luego de la caída de los precios de las commodities, y la inflexibilidad en el salario nominal amplifica el efecto negativo del shock en la economía.

\subsection{Los supuestos en los modelos CGE}

Los modelos de equilibrio general computable en términos operativos pueden diferenciarse por dos caminos, y de acuerdo a los diferentes supuestos utilizados los resultados pueden variar sensiblemente ${ }^{1}$. El primero de estos involucra las ecuaciones de comportamiento, que definen como se comportan los agentes en el modelo. El

\footnotetext{
${ }^{1} \mathrm{Al}$ respecto, Taylor (2006) realiza una crítica a los supuestos utilizados por el Banco Mundial para los modelos CGE para países en vías de desarrollo, y muestra como un mismo shock (liberalización comercial) puede tener diferentes consecuencias y recomendaciones de política de acuerdo a los supuestos utilizados.
} 
segundo refiere a los cierres macroeconómicos, que establecen los procesos de ajuste de los mercados ante cambios exógenos.

Las ecuaciones de comportamiento entre los modelos estándar y estructuralistas difieren en cómo se modelan los sectores productivos y en algunos aspectos de determinación de la demanda. En general, los modelos estándar utilizan funciones de producción que permiten cierto grado de sustitución entre factores, mientras que los estructuralistas se inclinan hacia tecnologías de coeficientes fijos (Taylor, 1990). Un rasgo distintivo del modelado estructuralista es que el capital se encuentra funcionando con capacidad ociosa, y de no definir restricciones en la capacidad de utilización, este supuesto es equivalente a suponer oferta horizontal del capital. Otro aspecto importante de diferenciación consiste en la determinación del precio final de los bienes. Si bien dentro de los modelos estándar existe literatura que modela mercados con competencia imperfecta (Harris, 1984) usualmente estos suponen que los productores no tienen poder de mercado. Al contrario, los estructuralistas generalmente determinan el precio aplicando un margen sobre los costos variables de producción, suponiendo que las firmas tienen poder para influir sobre los precios de mercado.

Así, el mark up es una de las principales diferencias entre los modelos. Al definirse la relación entre el precio y el costo variable, se está definiendo la distribución del ingreso entre el trabajo y el capital. La puja distributiva queda entonces expresada en el valor del mark up, que define cuáles serán los beneficios de las firmas. Esta variable, a diferencia de los modelos de competencia imperfecta, no surge de la interacción entre el costo marginal e ingreso marginal, sino que proviene directamente de los datos observados, sin establecer fundamentos microeconómicos (Taylor, 1990). Se supone que los acuerdos institucionales definen la distribución del ingreso, transformando la tasa de mark up en una variable exógena sobre la que se puede influir, en vez de una consecuencia inevitable de la estructura de mercado.

\subsection{Los cierres macroeconómicos}

Los modelos CGE incluyen mercados de factores y bienes e instituciones que participan en estos mercados, como los hogares, el gobierno, las empresas y el sector externo. Una diferencia central entre los modelos estructuralistas y estándar se produce en los denominados cierres macroeconómicos, esto es, cuales son los supuestos de funcionamiento y ajuste en los diferentes mercados (Robinson, 2006). Los modelos estándar utilizados para los países en vías de desarrollo, mantienen a grandes rasgos la estructura de ecuaciones de comportamiento utilizadas en los modelos neoclási- 
cos, e incorporan distintas rigideces, como el desempleo o la inmovilidad del capital, para captar las especificidades de las economías modeladas (Cicowiez 2011).

Por su parte, los modelos estructuralistas plantean diferentes supuestos para los mercados. En primera instancia, se alejan del supuesto de competencia perfecta al establecer que los precios finales se establecen a través de un margen sobre los costos variables. En el mercado de factores, suponen existencia de desempleo y capital específico, pero cuando se incorpora el supuesto de capacidad ociosa se permite variaciones en las cantidades de capital utilizada. De esta manera, la respuesta a los cambios en los precios internacionales, demanda, etc., se produce vía cantidades, aumentando o disminuyendo la producción mediante cambios en el mercado laboral. Manteniendo la estructura de supuestos básicos, es común encontrar modelos estructuralistas que incorporan sectores con formación de precios competitivos y que funcionan sin capacidad ociosa. (Serino, 2009).

Para modelar el sector externo, el ajuste puede producirse a través del tipo de cambio real o del ahorro del resto del mundo. No existe aquí una especificación única para los modelos estándar, pero es habitual suponer exógeno el ahorro del resto del mundo, siendo el tipo de cambio real la variable que ajusta para equilibrar el mercado, generando cambios en las cantidades exportadas e importadas. Los modelos estructuralistas suelen fijar el tipo de cambio nominal, ya que la consideran una herramienta de política, y el ajuste se produce mediante cambios en la cuenta corriente. La introducción de esta rigidez nominal conduce a que el tipo de cambio real no equilibre el sector externo, sino que sea el ahorro del resto del mundo la variable que ajusta ante shocks exógenos. Usualmente, los estructuralistas escogen el salario nominal como numerario, y como el tipo de cambio nominal también se encuentra fijo, el modelo es homogéneo cuando se modifican ambas variables simétricamente. Se pueden obtener resultados reales mediante variaciones en el salario o el tipo de cambio nominal, siempre que estos varíen en diferente proporción. En términos de política esto significa que se puede controlar al mismo tiempo el tipo de cambio y los salarios, dominando el pass through que podría generar una devaluación.

El cierre del sector gobierno puede realizarse de diferentes formas. Las variables que definen el déficit o superávit del gobierno son el gasto y el ingreso del sector público, y para afectar estas variables existen dos caminos. El primero consiste en endogeneizar el gasto real o las tasas impositivas, manteniendo constante el ahorro del gobierno. Este supuesto implica que ante shocks exógenos negativos el sector público 
automáticamente reduce su gasto o sube los impuestos ${ }^{2}$. El segundo camino supone que el gasto real y las alícuotas impositivas están fijas, siendo el ahorro la variable que reacciona ante cambios en la economía. En este caso, suponer que el gasto y las alícuotas son variables exógenas permite modelar medidas donde el gobierno tiene el control de la política fiscal, en vez de ser ésta una respuesta endógena del modelo. Por último, la forma en que se equilibra el canal ahorro-inversión es una diferencia sustancial entre los modelos. Los modelos estándar suelen suponer que la inversión es endógena, derivando en un modelo savings driven, mientras que los estructuralistas utilizan un cierre de tipo investment driven. Siguiendo la tradición keynesiana, estos últimos suponen que los empresarios definen sus planes de inversión de manera independiente del ahorro y si se producen shocks exógenos, la economía reacciona generando el ahorro necesario para financiar la inversión.

\subsection{Los supuestos en los modelos estándar y estructuralista}

Como marco general para ambos modelos se supuso que Argentina es un país pequeño en términos internacionales. Para el modelo estándar se supuso que todas las actividades se desempeñan en mercados competitivos, y fueron modeladas con funciones de producción CES (Elasticidad de Sustitución Constante). Existe desempleo y libre movilidad del factor trabajo entre sectores. El salario nominal es flexible y el salario real crece a medida que cae el desempleo. El capital es específico y la remuneración al capital varía entre actividades. El cierre del sector externo supone constante el ahorro del resto del mundo, y el canal de ajuste se realiza a través del tipo de cambio real. La inversión es endógena, y ajusta para equilibrarse con el ahorro disponible, por lo que el modelo es saving driven. El numerario utilizado es el índice de precios al consumidor.

El modelo estructuralista supone actividades con poder de mercado y capacidad ociosa y actividades que se desempeñan en mercados competitivos con utilización plena y eficiente de factores, de acuerdo a la tabla 1. Supone que trabajo es móvil entre sectores, hay desempleo en el mercado laboral, y el salario nominal y la tasa de mark up se fijan conforme a acuerdos institucionales. La inversión en la economía se define de manera independiente al ahorro, y es este último el que ajusta para financiar la inversión. El tipo de cambio nominal es considerado una herramienta de política, por lo que se fija de manera exógena. Así, el ahorro del resto del mundo

\footnotetext{
${ }^{2}$ Esta especificación no tiene en cuenta que los cambios en estas variables tienen consecuencias políticas que pueden dificultar este ajuste automático.
} 
es la variable que ajusta para equilibrar la cuenta corriente. Este cierre del sector externo permite evaluar la necesidad de financiamiento externo ante cambios en los precios internacionales, alícuotas impositivas, gasto público, entre otros parámetros de interés. En este modelo el salario es fijado como numerario.

Finalmente, el sector público y los hogares tienen la misma regla de cierre en los dos modelos. El gobierno define sus gastos y alícuotas impositivas de manera predeterminada, de manera que el ahorro del sector público se define endógenamente. Los hogares consumen y ahorran una porción constante de su ingreso, haciendo endógeno su ahorro.

\subsection{Especificaciones del modelo estructuralista}

Para definir las características del modelo estructuralista, se agruparon las actividades en cinco grandes grupos: aquellos que producen bienes primarios (PP), los que producen bienes manufacturados intensivos en uso de recursos naturales (MOA), los productores de bienes manufacturados de origen industrial (MOI), el sector de servicios (SS) y la administración pública (AP). Las actividades que producen bienes primarios, al igual que aquellas que son intensivas en uso de recursos naturales, definen sus precios de manera competitiva y los factores son utilizados de manera plena y eficiente. La administración pública, que solo utiliza trabajo en su función de producción, también tiene las características previamente descriptas. Estas actividades pertenecen al grupo con funciones de producción CES. Las actividades que producen bienes industriales y el sector servicios tienen poder de mercado, producen por debajo de su capacidad máxima y fueron modeladas de acuerdo a las especificaciones estructuralistas $^{3}$. En la siguiente tabla se definen las actividades junto a sus principales características:

\footnotetext{
${ }^{3}$ La clasificación de las actividades se realizó siguiendo a Serino (2009).
} 
Tabla 1: Descripción de las actividades y mercados

\begin{tabular}{ccc}
\hline Grupo & Actividades & Mercados \\
\hline \hline PP & 6 & $\begin{array}{r}\text { Producción con utilización plena de capital, } \\
\text { firmas competitivas }\end{array}$ \\
AP & 4 & $\begin{array}{r}\text { Producción con utilización plena de capital, } \\
\text { firmas competitivas }\end{array}$ \\
MOI & 1 & $\begin{array}{r}\text { Producción con utilización plena de capital, } \\
\text { firmas competitivas }\end{array}$ \\
SS & 9 & $\begin{array}{r}\text { Producción con capacidad ociosa, firmas } \\
\text { con poder de mercado }\end{array}$ \\
& 12 & $\begin{array}{r}\text { Producción con capacidad ociosa, firmas } \\
\text { con poder de mercado }\end{array}$ \\
\hline
\end{tabular}

Fuente: elaboración propia en base a Serino (2009)

\section{La estructura económica argentina}

\subsection{La Matriz de Contabilidad Social}

La aplicación de los modelos CGE se realiza construyendo en primera instancia una Matriz de Contabilidad Social (Social Accounting Matrix -SAM-), que aporta la base informativa para calibrar gran parte de los parámetros de comportamiento del modelo. Dicha matriz contiene la información de todos los intercambios que se realizan en la economía. Siguiendo a Chisari et al (2009), "La SAM reúne dos ideas importantes en economía. En primer lugar, la SAM generaliza la presentación de la matriz de insumo-producto para todas las transacciones de una economía. La otra idea importante se deriva de los principios de la contabilidad nacional y es que los ingresos siempre igualan a los gastos a nivel agregado". La SAM es una extensión de la Matriz Insumo-Producto Leontief, en donde en su marco más general incorpora los flujos de pagos a las familias, empresas, gobierno y resto del mundo, a la vez que registra la demanda de bienes y servicios realizada por estas instituciones (Robinson, 2006). En la siguiente tabla se presenta la Macro SAM construida para calibrar el modelo para Argentina correspondiente al año 2012. 
Tabla 2: MacroSAM Argentina en miles de millones de pesos, año 2012

\begin{tabular}{|c|c|c|c|c|c|c|c|c|c|c|c|c|c|c|}
\hline & act & com & f-lab & f-cap & $\operatorname{tax}$ & t-exp & cssoc & t-dir & hhd & gov & row & inv & dstk & total \\
\hline act & & 4,402 & & & & & & & & & & & & 4,402 \\
\hline com & 2,031 & & & & & & & & 1,829 & 417 & 428 & 474 & -3 & 5,175 \\
\hline f-lab & 1,302 & & & & & & & & & & 1 & & & 1,303 \\
\hline f-cap & 1,070 & & & & & & & & & & 11 & & & 1,081 \\
\hline $\operatorname{tax}$ & & 332 & & & & & & & & & & & & 332 \\
\hline t-exp & & 61 & & & & & & & & & & & & 61 \\
\hline $\operatorname{cssoc}$ & & & 180 & & & & & & & & & & & 180 \\
\hline t-dir & & & & & & & & & 223 & & & & & 223 \\
\hline hhd & & & 1,122 & 978 & & & & & 575 & 430 & 4 & & & 3,110 \\
\hline gov & & & & 34 & 332 & 61 & 180 & 223 & 35 & & 8 & & & 873 \\
\hline row & & 379 & 1 & 69 & & & & & 14 & 1 & & & & 464 \\
\hline sav & & & & & & & & & 433 & 26 & 11 & & & 470 \\
\hline dstk & & & & & & & & & & & & -3 & & -3 \\
\hline total & 4,402 & 5,175 & 1,303 & 1,081 & 332 & 61 & 180 & 223 & 3,110 & 873 & 464 & 470 & -3 & \\
\hline
\end{tabular}

Las cuentas de la SAM son: actividades -act-,productos -com-, trabajo -lab-, capital -cap-, impuestos -tax-, retenciones a las exportaciones -tax-exp- contribuciones a la seguridad social -csspc-, impuestos directos -tax-dir-, hogares -hhd-, gobierno -gov-, resto del mundo -row-, ahorro inversión -sav-inv-, variación de existencias -dstk-. Fuente: elaboración propia en base a INDEC, MECON, FMI

Si se lee la SAM en sentido vertical, las columnas expresan los pagos de las cuentas, mientras que si se lee en sentido horizontal, en las filas se encuentran las fuentes de las mismas. A modo de ejemplo, si se toma la primera columna y se lee de arriba hacia abajo, esta detalla los pagos que las actividades realizan a los productos, al trabajo y al capital. Si se toma la novena fila y se lee de izquierda a derecha, se encuentran las fuentes de ingresos de los hogares, que corresponden a los factores trabajo y capital, y las transferencias que el gobierno y el resto del mundo realizan a las familias. La SAM desagregada (micro SAM) utilizada para la calibración de los modelos, incluye 32 actividades y 32 productos. Además se tienen 5 tipos de impuestos (impuesto a las ventas, impuestos directos a los hogares, retenciones y aranceles al comercio exterior, aportes y contribuciones a la seguridad social), 3 instituciones (hogares, gobierno y resto del mundo) y 2 factores productivos (capital y trabajo). Las importaciones y los impuestos internos fueron distribuidos proporcionalmente entre las actividades y las instituciones demandantes. En la tabla 3 se presentan los principales indicadores sectoriales para el año 2012, base para la calibración del modelo: 
Tabla 3: Estructura Sectorial Argentina, año 2012

\begin{tabular}{|c|c|c|c|c|c|c|}
\hline & VA & PRD shr & EXP shr & EXP-OUT shr & IMP- shr & IMP-DEM shr \\
\hline Bienes & 36.2 & 47.0 & 85.5 & 17.7 & 81.8 & 15.4 \\
\hline Agropecuario & 6.5 & 6.2 & 16.1 & 25.4 & 0.6 & 1.2 \\
\hline Minería & 1.9 & 1.1 & 5.0 & 44.5 & 1.4 & 16.2 \\
\hline Hidrocarburos & 2.3 & 2.0 & 2.8 & 13.8 & 4.8 & 19.3 \\
\hline Electricidad & 2.8 & 2.6 & 0.2 & 0.6 & 0.5 & 1.5 \\
\hline Alimentos & 3.5 & 8.1 & 26.3 & 31.7 & 1.5 & 2.3 \\
\hline Bebidas y tabaco & 1.3 & 1.8 & 1.8 & 10.1 & 0.2 & 1.1 \\
\hline Textil & 1.6 & 1.9 & 2.9 & 14.5 & 3.1 & 13.9 \\
\hline Papel & 1.0 & 1.1 & 0.7 & 6.1 & 1.6 & 11.3 \\
\hline Refinación petroleo & 0.6 & 2.3 & 4.3 & 18.6 & 5.0 & 19.0 \\
\hline Quimicos & 2.0 & 3.4 & 7.5 & 21.6 & 14.4 & 31.9 \\
\hline Plásticos & 0.8 & 1.1 & 0.9 & 8.3 & 2.5 & 17.6 \\
\hline Minerales no metálicos & 0.6 & 0.8 & 0.3 & 3.9 & 0.7 & 7.6 \\
\hline Otras manufacturas & 0.6 & 0.5 & 0.1 & 2.7 & 1.2 & 16.8 \\
\hline Metálicos básicos & 1.1 & 0.2 & 0.1 & 5.9 & 0.5 & 19.5 \\
\hline Productos metálicos & 0.9 & 2.4 & 2.9 & 11.9 & 4.7 & 16.0 \\
\hline Maquinaria & 1.4 & 2.4 & 2.8 & 11.3 & 23.8 & 48.9 \\
\hline Vehículos & 1.0 & 1.8 & 10.3 & 54.3 & 14.7 & 60.1 \\
\hline Resto & 0.8 & 0.5 & 0.2 & 8.9 & 0.4 & 36.6 \\
\hline Construcción & 5.6 & 6.8 & 0.1 & 0.1 & 0.2 & 0.2 \\
\hline Servicios & 63.8 & 53.0 & 14.5 & 2.7 & 18.2 & 3.0 \\
\hline Comercio & 12.7 & 9.0 & 0.0 & 0.0 & 0.0 & 0.0 \\
\hline Hoteles y restaurantes & 1.8 & 2.2 & 2.7 & 11.9 & 4.9 & 17.9 \\
\hline Transporte & 4.4 & 5.0 & 3.1 & 6.0 & 4.0 & 6.7 \\
\hline Financieros & 3.4 & 3.2 & 0.0 & 0.0 & 0.2 & 0.7 \\
\hline Otros servicios & 19.3 & 17.4 & 8.5 & 4.8 & 8.6 & 4.3 \\
\hline Comunicaciones & 2.0 & 1.7 & 0.2 & 1.2 & 0.5 & 2.4 \\
\hline Administración pública & 7.6 & 5.8 & 0.0 & 0.0 & 0.0 & 0.0 \\
\hline Educación & 6.6 & 4.0 & 0.0 & 0.0 & 0.0 & 0.0 \\
\hline Salud & 5.7 & 4.3 & 0.0 & 0.0 & 0.0 & 0.0 \\
\hline Resto & 0.3 & 0.3 & 0.0 & 0.0 & 0.0 & 0.0 \\
\hline Total & 100.0 & 100.0 & 100.0 & 9.7 & 100.0 & 8.7 \\
\hline
\end{tabular}

Nota: VA: Valor Agregado Sectorial/ Valor Agregado Total; PRD shr: Producto sectorial/Producto Total; EXP shr: Exportaciones sectoriales/Exportaciones Totales; EXP-OUT shr: Exportaciones sectoriales/ Producto sectorial; IMP shr: Importaciones sectoriales/Importaciones Totales; IMP-DEM shr: Importaciones Sectoriales/Demanda sectorial.

Fuente: elaboración propia en base a INDEC. 
La primer columna de la tabla precedente detalla la composición del valor agregado a nivel sectorial. Allí se observa que los servicios producen el $63.8 \%$ del total, mientras que los bienes producen el $36.2 \%$ restante. Dentro de estos últimos, el sector agropecuario genera el $6.5 \%$ del valor agregado total, siendo el sector de mayor peso dentro de la producción de bienes, mientras que la industria alimenticia ocupa el segundo lugar, con un 3.5\% del valor agregado. La importancia de los bienes intensivos en recursos naturales en el producto puede verse en la segunda columna. En términos del producto total, la producción agropecuaria representa el $6.2 \%$, la extracción de hidrocarburos el $2 \%$, y la minería equivale al 1.1\%. La manufacturas de origen agropecuario, como la producción de alimentos y las bebidas y tabaco equivalen el $8.1 \%$ y el $1.8 \%$ respectivamente. En total estos sectores tienen un peso en el producto del $19.2 \%$, y representan el $62.7 \%$ de los bienes producidos. Este patrón se repite al analizar la composición de las ventas al exterior. En la columna 3 se presentan las exportaciones sectoriales en términos del total exportado. El sector de alimentos (26.3\%), el sector agropecuario (16.1\%), la minería (5\%), los hidrocarburos (2.8\%), y los alimentos y bebidas (1.8\%), explican el $52 \%$ de las exportaciones. Para analizar la intensidad exportadora de la producción sectorial, en la columna 4 se presenta el ratio exportaciones sobre el valor bruto de la producción (VBP) por sector. En este caso, el sector automotriz lidera el ranking de exportaciones en términos del producto sectorial, ya que exporta el $54.3 \%$ de su producción. Le siguen la minería (44.5\%), el sector alimenticio (31.7\%), y el sector agropecuario $(25.4 \%)$.

Las últimas dos columnas permiten analizar las importaciones en términos del total importado y del consumo sectorial. Aquí se observa el elevado protagonismo que los sectores industriales tienen en las importaciones argentinas. Analizando la quinta columna se puede apreciar que los principales productos importados corresponden al sector de maquinaria $23.8 \%$, automotriz $14.7 \%$, químicos $14.4 \%$, y productos metálicos $(4.7 \%)$. En conjunto estos sectores representan el $57.6 \%$ del total de importaciones. La sexta columna detalla las importaciones en relación al consumo por sector. Una vez más, la importación automotriz se encuentra al tope de la lista, ya que importa el $60.1 \%$ del total del consumo sectorial ${ }^{4}$. Le siguen el sector de maquinaria, con $48.9 \%$, los productos químicos, con $31.9 \%$, los metálicos básicos con $19.5 \%$, los productos plásticos con $17.6 \%$, productos metálicos con $16 \%$, y otras

\footnotetext{
${ }^{4} \mathrm{La}$ intensidad de exportaciones e importaciones de la industria automotriz se explican por el régimen de intercambio compensado entre Brasil y Argentina. Gran parte del comercio entre estos países se realiza a nivel intraindustria.
} 
manufacturas con $16.8 \%$. Por último, cabe realizar una consideración respecto al sector de hidrocarburos. Al año 2012, la importación de gas y petróleo representaron el $19.3 \%$ del consumo sectorial, y los combustibles y lubricantes importados alcanzaron el $19 \%$ del total consumido.

En síntesis, la tabla 3 muestra el elevado protagonismo que los productos intensivos en recursos naturales tienen en el producto y en las exportaciones, a la vez que refleja el alto peso de las importaciones industriales en la economía. En consecuencia, los cambios en la demanda externa y/o en los precios internacionales tienen un elevado impacto en el conjunto de la economía argentina.

\section{Los modelos estándar y estructuralista}

\subsection{Ecuaciones principales}

En esta sección se presentan las principales ecuaciones que distinguen ambos modelos (en el apéndice se encuentran los modelos completos ${ }^{5}$ ), con sectores cuya producción está definida de manera neoclásica y sectores que se modelan al estilo estructuralista. Esta especificación permite cambiar los supuestos sobre el comportamiento de los mercados individuales. En el caso extremo, se puede considerar que todas las actividades tienen poder de mercado, o al contrario, suponer que todas se encuentran en competencia perfecta.

Las actividades modeladas al estilo neoclásico, utilizan funciones de producción que permiten sustitución entre los factores. Para este tipo de actividades se utilizó una función de elasticidad de sustitución constante (CES), de la siguiente forma:

$$
Q V A_{a}=\pi_{a} \sum_{c}\left[\delta_{f, a}^{v a} Q F_{f, a}^{-\rho v a_{a}}\right]^{-\frac{1}{\rho v a_{a}}}
$$

La cantidad de valor agregado $Q V A_{a}$ surge de la combinación de los factores $Q F_{f, a}$ de acuerdo a los parámetros de proporción $\delta_{f, a}^{v a}$, el factor de productividad $\pi_{a}$ y la elasticidad de sustitución $\sigma v a_{a}=\frac{1}{\rho v a_{a}+1}$.

La demanda de factores se obtiene de resolver las condiciones de primer orden del problema de minimización de costos:

\footnotetext{
${ }^{5}$ La cantidad de ecuaciones de los modelos son 55 para el modelo estándar y 53 para el modelo estructuralista. Luego de la calibración dichas ecuaciones ascienden a 6448 y 6446 respectivamente.
} 


$$
\frac{Q F_{f, a}}{Q V A_{a}}=\left(\frac{P V A_{a}}{W F_{f} W F D I S T_{f, a}}\right)^{\sigma v a_{a}}\left(\delta_{f, a}^{v a}\right)^{\sigma v a_{a}} \pi_{a}^{\left(\sigma v a_{a}-1\right)}
$$

Donde $W F D I S T_{f, a}$ es la distorsión del precio factorial. Dicho diferencial permite asignar distintas remuneraciones factoriales entre actividades ${ }^{6}$. Las actividades demandan bienes intermedios en proporciones fijas, y el valor total de la producción de las actividades surge de combinar en proporciones fijas el valor agregado proveniente de la función de producción y los bienes intermedios.

Para el caso de las actividades de carácter estructuralista, la tecnología de producción utilizada es Leontief, dada por la función de producción $\min \left\{\frac{Q F_{f, a}}{b_{f, a}}\right\}$. La demanda de trabajo utiliza el coeficiente fijo $b_{l a b, a}$, quedando expresado de la siguiente manera:

$$
b_{l a b, a}=\frac{Q F_{l a b, a}}{Q A_{a}}
$$

Donde $Q A_{a}$ expresa las cantidades producidas por cada actividad. Con respecto al capital, en los modelos estructuralistas la ecuación de "demanda" de capital se establece a través de la capacidad de utilización $U_{c a p, a}$. Como las actividades se encuentran produciendo con capacidad ociosa, no hay pleno empleo de capital, y la respuesta ante cambios en la demanda se produce aumentando o disminuyendo la utilización de la capacidad instalada. En el presente modelo no se establecen límites a la capacidad de utilización, lo que es equivalente a suponer que el capital tiene oferta horizontal y la tasa de mark up es siempre exógena. Si aumenta la demanda agregada, los empresarios responden contratando más mano de obra y aumentando la utilización del capital.

$$
U_{c a p, a}=\frac{Q A_{a}}{Q F_{c a p, a}}
$$

Los precios de los factores $W F_{f}$ se definen por dos caminos diferentes: el salario está dado por la configuración institucional, y el precio del capital se deriva de la

\footnotetext{
${ }^{6} \mathrm{El}$ precio de los factores está dado por $W F_{f} W F D I S T_{f, a}$. Para modelar capital específico y distintos pagos al capital, se supone que $W F_{\text {cap }}$ se encuentra fijo, permitiendo variar $W F D I S T_{\text {cap }, a}$ por actividad. En el caso del trabajo, hay una sola remuneración factorial, por lo tanto $W F_{l a b}$ es flexible y $W F D I S T_{l a b, a}$ es constante.
} 
tasa de mark up $T A O_{a}$ y la capacidad de utilización ${ }^{7}$ :

$$
W F_{f} W F D I S T_{f, a}=P A_{a} \frac{T A O_{a} U_{c a p, a}}{\left(1+T A O_{a}\right)\left(1+t a_{a}\right)}
$$

El precio de las actividades con poder de mercado se define a través del margen que las empresas establecen sobre su costo variable $C V_{a}$. El mismo está compuesto por la cantidad de trabajo por unidad producida $b_{l a b, a}$, y la cantidad de bienes intermedios por unidad producida $i c a_{c, a}$, ambos expresados en términos nominales.

$$
C V_{a}=\sum_{l a b} W F_{l a b} W F D I S T_{l a b, a}+\sum_{c} i c a_{c, a} P Q D_{c, a}
$$

El margen sobre el costo variable $C V_{a}$ implica beneficios positivos para las firmas. En los modelos estructuralistas el mark up surge de la observación de los datos, esto es, una consecuencia empírica que surge de dividir el flujo de beneficios observado por el costo variable. El supuesto que subyace es que el mark up viene dado de acuerdo a la configuración institucional, que establece una determinada distribución del ingreso entre el capital y el trabajo. Esto tiene consecuencias directas en la política económica, ya que el mark up no es función del poder de mercado, y puede modificarse a través de negociaciones que conduzcan a nuevos arreglos institucionales. El precio de las actividades con mark up se definen de la siguiente manera:

$$
P A_{a}=\left(1+T A O_{a}\right)\left(1+t a_{a}\right) C V_{a}
$$

Donde $t a_{a}$ es la tasa impositiva sobre las actividades. Para modelar la demanda de importaciones se utilizó el enfoque propuesto por Armington (1969) que a través de una función CES supone sustitución imperfecta entre la demanda doméstica $Q D_{c, a c}$ y la importada $Q M_{c, a c}$. Esta forma particular permite modelar el comercio internacional intra industrial, al diferenciar el consumo por país de origen. En el modelo, cada institución demandante tiene su propia función CES dada por la ecuación 17,

\footnotetext{
${ }^{7}$ Para $t a_{a}=0$ y $W F D I S T_{f, a}=1$ se tiene:

$$
\begin{array}{r}
W F_{c a p} Q F_{c a p}=P A_{a} Q A_{a}-C V_{a} Q A_{a} \\
W F_{c a p} Q F_{c a p}=\left(P A_{a}-C V_{a}\right) Q A_{a} \\
W F_{c a p} Q F_{c a p}=C V_{a} T A O_{a} Q A_{a} \\
\left(1+T A O_{a}\right) W F_{c a p} Q F_{c a p}=\left(1+T A O_{a}\right) C V_{a} T A O_{a} Q A_{a} \\
W F_{f}=P A_{a} \frac{T A O_{a} U_{c a p, a}}{\left(1+T A O_{a}\right)\left(1+t a_{a}\right)}
\end{array}
$$
}


construyendo el bien compuesto $Q Q_{c, a c}$, de manera que se pueden suponer diferentes elasticidades de sustitución de acuerdo a las características particulares de cada agente $^{8}$. La condición de tangencia que surge de las condiciones de primer orden del problema de minimización de gasto determina las cantidades consumidas de cada bien y se define en la ecuación 18, mientras que la ecuación 10 especifica el precio de demanda del bien compuesto como el promedio ponderado entre bienes domésticos e importados.

$$
\begin{gathered}
Q Q_{c, a c}=a q_{c, a c}\left[\delta q_{c, a c}^{M} Q M_{c, a c}^{-\rho q_{c, a c}}+\delta q_{c, a c}^{D} Q D_{c, a c}^{-\rho q_{c, a c}}\right]^{-\frac{1}{\rho q_{c, a c}}} \\
\frac{Q M_{c, a c}}{Q D_{c, a c}}=\left(\frac{P D_{c}}{P M_{c, a c}} \frac{\delta q_{c, a c}^{M}}{\delta q_{c, a c}^{D}}\right)^{\frac{1}{\left(1+\rho q_{c, a c}\right)}} \\
P Q S_{c, a c} Q Q_{c, a c}=P M_{c, a c} Q M_{c, a c}+P D_{c, a c} Q D_{c, a c} \\
P Q D_{c, a c}=P Q S_{c, a c}\left(1+t q_{c, a c}\right)
\end{gathered}
$$

La ecuación 11 incorpora el impuesto a las ventas, estableciendo una diferencia entre el precio de oferta $P Q S_{c, a c}$ y el precio de demanda $P Q D_{c, a c}$.

El precio doméstico de los bienes importados y exportados está definido en las siguientes ecuaciones:

$$
\begin{gathered}
P M_{c, a c}=\left(1+t m_{c, a c}\right) E X R p w m_{c} \\
P E_{c}=\left(1+t e_{c}\right) E X R p w e_{c}
\end{gathered}
$$

Donde $p w e_{c}$ y $p w m_{c}$ expresan los precios internacionales en moneda extranjera, $t m_{c, a c}$ y $t e_{c}$ indican las tasas arancelarias y de retenciones, y $E X R$ es el tipo de cambio nominal.

Para modelar el destino de la producción nacional se utilizó una función de transformación de elasticidad constante CET, de manera de incluir la posibilidad de orientar la oferta al mercado interno o al exterior, de acuerdo a las variaciones en el tipo de cambio real de cada bien. La función $\mathrm{CET}^{9}$ se presenta en la ecuación 14, y la con-

\footnotetext{
${ }^{8}$ La función CES vincula la demanda doméstica $Q D_{c, a c}$ con la importada $Q M_{c, a c}$ de acuerdo a los parámetros de proporción $\delta q_{c, a c}^{M}$ y $\delta q_{c, a c}^{D}$, de escala $a q_{c, a c}$ y de elasticidad $\rho q_{c, a c}$

${ }^{9}$ La función CET vincula la oferta al mecado doméstico $Q D S_{c, a c}$ y al exterior $Q E_{c, a c}$ de acuerdo
} 
dición de beneficio nulo para el productor del bien c se expresa en la ecuación 15. De las condiciones de primer orden de maximización de beneficios surge la ecuación 16, que combinadas con las ecuaciones precedentes establecen la oferta de bienes al mercado doméstico y al exterior.

$$
\begin{gathered}
Q X_{c}=a t_{c}\left[\delta t_{c}^{E} Q E_{c}^{\rho t_{c}}+\delta t_{c}^{D} Q D S_{c}^{\rho t_{c}}\right]^{\frac{1}{\rho t_{c}}} \\
P X_{c} Q X_{c}=P D S_{c} Q D S_{c}+P E_{c} Q E_{c} \\
\frac{Q E_{c}}{Q D S_{c}}=\left(\frac{P E_{c}}{P D_{c}} \frac{\delta t_{c}^{D}}{\delta t_{c}^{E}}\right)^{\frac{1}{\left(\rho t_{c}-1\right)}}
\end{gathered}
$$

El tipo de cambio real agregado se define como el ratio entre el tipo de cambio nominal y el índice de precios internos del productor DPI:

$$
\begin{gathered}
\sum_{c} d w t s_{c} P D_{c}=D P I \\
R E X R=\frac{E X R}{D P I}
\end{gathered}
$$

En ambos modelos se supuso que el mercado laboral funciona con desempleo. En el modelo estructuralista no se impusieron restricciones a la contratación de trabajadores, lo que implica que la oferta es horizontal para el tramo relevante. En el modelo estándar el desempleo fue modelado introduciendo una wage curve, que relaciona el salario real $W F R E A L_{l a b}$ definido en la ecuación 19 y la tasa de desempleo $U E R A T_{l a b}$ de manera negativa a través de la ecuación $20^{10}$ :

$$
\begin{gathered}
W F R E A L_{l a b}=\frac{W F_{l a b}}{C P I} \\
\frac{W F R E A L_{l a b}}{\overline{W F R E A L 0_{l a b}}}-1=- \text { phillips }_{l a b}\left(\frac{U E R A T_{l a b}}{\overline{U E R A T 0_{l a b}}}-1\right) \\
Q F S_{l a b}\left(1-U E R A T_{l a b}\right)=\sum_{a} Q F_{l a b, a}
\end{gathered}
$$

La ecuación 21 establece la condición de equilibrio en el mercado laboral con desem-

$\overline{\text { a los parámetros de proporción } \delta t_{c}^{D} \text { y } \delta} t_{c}^{E}$, de escala $a t_{c}$ y de elasticidad $\rho t_{c}$

${ }^{10} \mathrm{El}$ valor utilizado para el parámetro phillips $s_{l a b}$ es 0.1, de acuerdo a la estimación para Argentina en Cicowiez y Mercado (2009) 
pleo, donde $Q F S_{l a b}$ es la oferta total del factor trabajo (Cicowiez y Mercado, 2009). Finalmente, En el presente modelo hay cuatro conjuntos de elasticidades: las que pertenecen a las funciones Armington, las correspondientes a las funciones de transformación CET, aquellas utilizadas para las funciones de producción CES, y las que corresponden a la función de utilidad Cobb Douglas. Para las funciones de producción CES y las funciones de oferta CET se utilizaron las elasticidades estimadas en Cicowiez $(2011)^{11}$. Las cuasi-elasticidades de la función de utilidad fueron obtenidas directamente de la SAM, mientras que para las elasticidaddes de sustitución de las funciones Armington se asumieron valores de 0.1 para las actividades y 1.5 para los hogares, el gobierno y la inversión. De esta manera, se supone que es prácticamente nula la posibilidad de sustitución de productos importados por domésticos en los insumos intermedios, al contrario de las instituciones, que tienen mayor capacidad de sustitución ante cambios en las variables exógenas.

\section{Simulaciones}

Con el fin de analizar la vulnerabilidad de la Argentina ante cambios en los principales productos de exportación y los resultados que se obtienen al utilizar diferentes supuestos micro y macroeconómicos, se simuló una caída del $25 \%$ en los precios internacionales de los productos agropecuarios y de alimentos. Este shock fue aplicado a los dos modelos construidos. Posteriormente se evaluó el impacto de una reducción del $50 \%$ en las retenciones a las exportaciones de dichos productos. Por último, se simuló con el modelo estructuralista una devaluación del $10 \%$, con el objetivo de atenuar el efecto negativo del shock exógeno inicial. En resumen, los escenarios simulados fueron:

1. Escenario pwagr: reducción del $25 \%$ de los precios internacionales de exportación (pwe) e importación (pwm) de los productos agropecuarios y de alimentos.

2. Escenario pwagr-ret: Disminución del $50 \%$ de las retenciones a las exportaciones aplicadas a los productos agropecuarios y alimentos luego del shock inicial.

3. Escenario pwagr-dev: Devaluación del $10 \%$ del tipo de cambio nominal después del shock, para el modelo estructuralista.

\footnotetext{
${ }^{11}$ En el anexo se presentan las elasticidades utilizadas para la calibración.
} 
Los resultados agregados se presentan en la siguiente tabla:

Tabla 4: Resultados agregados, en términos reales (cambio \% respecto de la base)

\begin{tabular}{|c|c|c|c|c|c|c|}
\hline & \multirow[b]{2}{*}{ Base (miles mm $\$$ ) } & \multicolumn{2}{|c|}{ Estándar } & \multicolumn{3}{|c|}{ Estructuralista } \\
\hline & & pwagr & pwagr-ret & pwagr & pwagr-ret & pwagr-dev \\
\hline PBI & 2496.88 & $-0.5 \%$ & $-0.4 \%$ & $-2.7 \%$ & $-1.6 \%$ & $0.9 \%$ \\
\hline Consumo de los hogares & 1655.34 & $-1.4 \%$ & $-1 \%$ & $-2.8 \%$ & $-1.6 \%$ & $-0.1 \%$ \\
\hline Exportaciones & 366.8 & $1.9 \%$ & $3.5 \%$ & $-7.7 \%$ & $-4.4 \%$ & $3.3 \%$ \\
\hline Importaciones & 379.31 & $-6.1 \%$ & $-5.4 \%$ & $-2.2 \%$ & $-1.1 \%$ & $-3.2 \%$ \\
\hline Inversión & 452.17 & $-6.6 \%$ & $-8.1 \%$ & 0 & 0 & 0 \\
\hline Cambio en Ahorro ROW & 0 & 0 & 0 & 54.638 & 49.843 & 8.182 \\
\hline Tipo de cambio real (n índice) & 1 & $11.4 \%$ & $9.1 \%$ & $1.8 \%$ & $1.1 \%$ & $9.7 \%$ \\
\hline Desempleo (en \% del total) & $7.2 \%$ & $7.8 \%$ & $7.8 \%$ & $9.8 \%$ & $8.8 \%$ & $6.4 \%$ \\
\hline Salario real (n índice) & 1 & $-0.8 \%$ & $-0.7 \%$ & $1.7 \%$ & $1.2 \%$ & $-0.8 \%$ \\
\hline
\end{tabular}

Fuente: resultados del modelo

Como se observa en la tabla 4 en la segunda y cuarta columna, la caída en los precios internacionales de los principales productos de exportación genera resultados sensiblemente distintos en las principales variables macroeconómicas de acuerdo al modelo utilizado.

En el modelo estándar, el desequilibrio inicial en la cuenta corriente producto del shock se resuelve con una depreciación del tipo de cambio real del 11.4\%. Este ajuste cambiario se realiza de manera automática para mantener constante el saldo de la cuenta corriente. La devaluación real orienta la producción nacional al sector externo, y las exportaciones crecen un $1.9 \%$. A su vez, las importaciones se encarecen, y se reducen las cantidades demandadas en $-6.1 \%$. En suma, este cierre del sector externo permite amortiguar el efecto negativo del shock en el nivel de actividad, debido a que el aumento del tipo de cambio real aumenta las exportaciones y provoca la sustitución de importaciones por productos domésticos. No obstante, el PBI se contrae $-0.5 \%$, el desempleo aumenta 0.6 puntos porcentuales y el salario real cae $-0.8 \%$ luego de la reasignación factorial ${ }^{12}$. Los ingresos agregados de capital y trabajo disminuyen, reduciendo el ingreso (y ahorro) de los hogares. El sector público

\footnotetext{
${ }^{12}$ Las actividades que incrementan su demanda aumentan la producción contratando más trabajadores, y debido al capital específico, se reduce la productividad marginal del trabajo.
} 
también reduce sus ingresos, debido a la menor recaudación por retenciones a las exportaciones y la menor recaudación vinculada al nivel de actividad, y su ahorro cae un $-49 \%$. Como consecuencia, el ahorro agregado disminuye y la inversión retrocede un $-6.6 \%$.

En el modelo estructuralista la disminución de los precios de los bienes agropecuarios y de alimentos impacta de lleno en la balanza comercial. Debido a que el tipo de cambio nominal es una variable de política y se fija de manera exógena, el ajuste ante la caída del precio internacional se produce mediante un aumento del ahorro externo. La caída de las exportaciones por encima de las importaciones aumenta el ahorro del resto del mundo en 54.638 millones, y el ahorro interno se reduce para mantener constante la inversión. El mecanismo de ajuste se produce mediante la caída en el nivel de actividad, que reduce el ahorro de los hogares y del gobierno. Los menores precios externos disminuyen la producción de bienes exportados, reduciendo la demanda laboral, el empleo y finalmente el nivel de actividad en $-2.7 \%$. La baja en la demanda agregada aumenta el desempleo hasta el 9.8\%, mientras que el descenso del índice de precios al consumidor aumenta el salario real en $1.7 \%$. El efecto final sobre el ingreso de los hogares es negativo, y éstos disminuyen su consumo, potenciando el efecto contractivo del shock inicial. La caída de la demanda agregada repercute en las finanzas públicas disminuyendo la recaudación impositiva, y junto a las menores percepciones por retenciones a las exportaciones contraen el ingreso total del gobierno.

De esta manera, el ajuste del sector externo y el equilibrio del canal ahorro-inversión son cruciales en los resultados obtenidos. En el modelo estándar, el ajuste automático del tipo de cambio real deriva en un aumento de las exportaciones no agroalimentarias, disminución de las importaciones, y una leve contracción del PBI. En el modelo estructuralista, al no producirse ajustes endógenos en el tipo de cambio se amplifican los efectos negativos en el nivel de actividad de la caída en los precios internacionales. Adicionalmente, el cierre estructuralista permite cuantificar la necesidad de financiamiento externo para mantener constante la inversión, o dicho de otro modo, los requerimientos de importaciones para sostener el funcionamiento de la economía sin afectar el tipo de cambio nominal. En la práctica, el aumento del ahorro del resto del mundo puede cubrirse con financiamiento externo o con disminución de reservas internacionales.

En el modelo estándar la restricción externa se pone de manifiesto mediante la exogeneidad del ahorro del resto del mundo. Al no contar con financiamiento externo, 
la economía debe generar sus propios ajustes para mantener constante la cuenta corriente. En el caso estructuralista la restricción externa no se encuentra activa, ya que el ahorro externo ajusta ante cambios en la demanda agregada. Si dicha restricción se tornase operativa, el ajuste en la economía se produciría a través de la disminución de la inversión, la baja del gasto público, o una combinación de ambas opciones. Al ser estas dos variables exógenas en el modelo, el gobierno tiene cierto margen para administrar el ajuste, pero en última instancia es necesario endogeneizar alguna de estas variables para alcanzar el equilibrio, y dicha elección dependerá de los arreglos institucionales entre empresarios, gobierno y familias. Una alternativa posible consiste en disminuir la inversión en una proporción fija y permitir que el gasto público se torne endógeno. Para graficar esta posibilidad, se simuló el mismo descenso en los precios internacionales del escenario pwagr, incorporando la restricción al financiamiento externo y reduciendo la inversión en un $-10 \%$. En este caso, el gasto público debería retroceder $-37.2 \%$ para que el ahorro disponible sea suficiente para financiar la inversión, y el PBI se contraería -14.8\% luego del shock inicial.

\subsection{Análisis del comercio internacional}

La siguiente tabla muestra los resultados de las simulaciones en las exportaciones de la economía: 
Tabla 5: Exportaciones, en términos reales (cambio porcentual respecto de la base)

\begin{tabular}{lcccccc}
\hline & & \multicolumn{2}{c}{ Estándar } & \multicolumn{3}{c}{ Estructuralista } \\
Productos exportados & Base (miles mm $\$)$ & pwagr & pwagr-ret & pwagr & pwagr-ret & pwagr-dev \\
\hline \hline Agropecuario & 53.219 & $-4.9 \%$ & $-1.5 \%$ & $-7.4 \%$ & $-3.4 \%$ & $-5.5 \%$ \\
Minería & 19.42 & $3.5 \%$ & $4.4 \%$ & $1.1 \%$ & $0.6 \%$ & $0.7 \%$ \\
Hidrocarburos & 5.482 & $18.5 \%$ & $14.4 \%$ & $16.3 \%$ & $9.7 \%$ & $13.5 \%$ \\
alimentos & 89.434 & $-18.1 \%$ & $-8.2 \%$ & $-26.1 \%$ & $-15.5 \%$ & $-19.9 \%$ \\
Bebidas y tabaco & 7.161 & $14.8 \%$ & $11.6 \%$ & $2 \%$ & $1.4 \%$ & $12.8 \%$ \\
Textil & 12.115 & $14 \%$ & $11 \%$ & $0.8 \%$ & $0.7 \%$ & $18.3 \%$ \\
Papel & 2.989 & $8.5 \%$ & $6.9 \%$ & $-1 \%$ & $-0.6 \%$ & $7.8 \%$ \\
Refinación petroleo & 11.041 & $4.3 \%$ & $3.5 \%$ & $1.2 \%$ & $0.8 \%$ & $2.6 \%$ \\
Quimicos & 29.619 & $7 \%$ & $5.7 \%$ & $-2 \%$ & $-1.2 \%$ & $10.7 \%$ \\
Plásticos & 3.917 & $10 \%$ & $8.1 \%$ & $-2.1 \%$ & $-1.3 \%$ & $11 \%$ \\
Minerales no metálicos & 1.275 & $8.7 \%$ & $6.8 \%$ & $-0.1 \%$ & $0 \%$ & $8.2 \%$ \\
Productos metálicos & 12.021 & $10.6 \%$ & $8.6 \%$ & $-0.7 \%$ & $-0.4 \%$ & $17.3 \%$ \\
Maquinaria & 11.512 & $7.6 \%$ & $5.7 \%$ & $-1.2 \%$ & $-0.7 \%$ & $10.7 \%$ \\
Vehículos & 42.805 & $5.4 \%$ & $4.4 \%$ & $-1.5 \%$ & $-0.9 \%$ & $18.1 \%$ \\
Hoteles y restaurantes & 11.386 & $24.8 \%$ & $19.5 \%$ & $2.1 \%$ & $1.7 \%$ & $26.8 \%$ \\
Transporte & 13.243 & $19.9 \%$ & $16 \%$ & $-1.9 \%$ & $-1.1 \%$ & $17.4 \%$ \\
Resto & 40.161 & $20.1 \%$ & $16.1 \%$ & $-2.7 \%$ & $-1.6 \%$ & $20.6 \%$ \\
\hline Total & 366.8 & $1.9 \%$ & $3.5 \%$ & $-7.7 \%$ & $-4.4 \%$ & $3.3 \%$ \\
\hline
\end{tabular}

Fuente: resultados del modelo

El ajuste del sector externo del cierre estándar conduce a un crecimiento generalizado de las exportaciones no agroalimentarias. El aumento del tipo de cambio real tiene un efecto expansivo que puede observarse en la segunda columna de la tabla 5 . A excepción de los productos agropecuarios y de alimentos, todos los productos aumentan sus cantidades exportadas. El sector más afectado por la disminución de precios es el de alimentos, ya que tiene relación exportación-producto del $31 \%$. Este sector baja sus exportaciones un -18.1\%. El sector agropecuario, de menor intensidad exportadora que la industria alimenticia (17\%), hace lo propio un $-4.9 \%$. Debido a la mayor elasticidad de transformación, la industria de agroalimentos puede orientar su producción hacia el mercado interno en mayor proporción que el sector agropecuario.

En el modelo estructuralista, el shock de precios (detallados en la cuarta columna) tiene efectos contrapuestos a nivel sectorial. Mientras que los sectores agropecuarios y de alimentos reducen sus cantidades exportadas, al igual que los productos 
industriales, el sector de extracción de hidrocarburos aumenta sus exportaciones un $16.3 \%$. Este incremento se produce por la elevada elasticidad de transformación de la oferta, que produce que la baja en la demanda interna libere saldos exportables que la industria vende al exterior. Lo mismo ocurre en menor medida con el sector de bebidas y tabaco, refinación de petróleo y minería, que aumentan sus exportaciones un $2 \%, 1.2 \%$ y $1 \%$ respectivamente. Para el resto de los productos, la caída en la actividad reduce las cantidades exportadas.

Con respecto a las importaciones, tanto en el modelo estándar como en el modelo estructuralista se registran caídas en las cantidades en los mismos productos. En la siguiente tabla se detallan los efectos de las simulaciones en los productos importados:

Tabla 6: Importaciones, en términos reales (cambio \% respecto de la base)

\begin{tabular}{|c|c|c|c|c|c|c|}
\hline \multirow[b]{2}{*}{ Productos importados } & \multirow[b]{2}{*}{ Base (miles mm \$) } & \multicolumn{2}{|c|}{ Estándar } & \multicolumn{3}{|c|}{ Estructuralista } \\
\hline & & pwagr & pwagr-ret & pwagr & pwagr-ret & pwagr-dev \\
\hline Agropecuario & 2.389 & $1 \%$ & $4.5 \%$ & $0.8 \%$ & $4.6 \%$ & $2.1 \%$ \\
\hline Minería & 5.147 & $-2.2 \%$ & $-3.1 \%$ & $-1.2 \%$ & $-0.7 \%$ & $0 \%$ \\
\hline Hidrocarburos & 18.148 & $-1.2 \%$ & $-0.9 \%$ & $-1.9 \%$ & $-1.1 \%$ & $-0.7 \%$ \\
\hline alimentos & 5.613 & $21.6 \%$ & $25.7 \%$ & $31.9 \%$ & $35.2 \%$ & $25.5 \%$ \\
\hline Bebidas y tabaco & 0.798 & $-13.8 \%$ & $-11.2 \%$ & $-5.3 \%$ & $-3.3 \%$ & $-10.8 \%$ \\
\hline Textil & 11.811 & $-10.2 \%$ & $-8.2 \%$ & $-4.3 \%$ & $-2.7 \%$ & $-7.9 \%$ \\
\hline Papel & 6.069 & $-4.3 \%$ & $-3.3 \%$ & $-3.8 \%$ & $-2.3 \%$ & $-2.5 \%$ \\
\hline Refinación petroleo & 19.002 & $-5.6 \%$ & $-4.5 \%$ & $-4 \%$ & $-2.5 \%$ & $-3.6 \%$ \\
\hline Quimicos & 54.655 & $-5.1 \%$ & $-4.1 \%$ & $-3.5 \%$ & $-2.1 \%$ & $-3 \%$ \\
\hline Plásticos & 9.511 & $-3 \%$ & $-2.5 \%$ & $-3 \%$ & $-1.8 \%$ & $-0.4 \%$ \\
\hline Minerales no metálicos & 2.739 & $-4.3 \%$ & $-5.2 \%$ & $-1 \%$ & $-0.6 \%$ & $-0.2 \%$ \\
\hline Productos metálicos & 17.716 & $-2.3 \%$ & $-3.1 \%$ & $-1.1 \%$ & $-0.7 \%$ & $3.9 \%$ \\
\hline Maquinaria & 90.366 & $-7.7 \%$ & $-7.7 \%$ & $-1.8 \%$ & $-1.1 \%$ & $-3.6 \%$ \\
\hline Vehículos & 55.882 & $-6.9 \%$ & $-6.6 \%$ & $-2.4 \%$ & $-1.5 \%$ & $-4 \%$ \\
\hline Hoteles y restaurantes & 18.444 & $-12.8 \%$ & $-10.4 \%$ & $-4.8 \%$ & $-3 \%$ & $-10.5 \%$ \\
\hline Transporte & 15.064 & $-8 \%$ & $-6.5 \%$ & $-3.7 \%$ & $-2.2 \%$ & $-5.8 \%$ \\
\hline Resto & 45.975 & $-7.7 \%$ & $-6.5 \%$ & $-3.3 \%$ & $-2 \%$ & $-5.4 \%$ \\
\hline Total & 379.329 & $-6.1 \%$ & $-5.4 \%$ & $-2.2 \%$ & $-1.1 \%$ & $-3.2 \%$ \\
\hline
\end{tabular}

Fuente: resultados del modelo

Si bien en ambos casos caen las importaciones, las causas que conducen a estos resultados difieren entre modelos. En el primer caso, la reducción de las importaciones se produce por el aumento en el tipo de cambio real que encarece estos produc- 
tos, mientras que en el modelo estructuralista la caída en las importaciones se debe principalmente a la retracción de la demanda agregada.

Los únicos productos que aumentan las cantidades importadas son los agropecuarios y los alimentos, y esto es debido a la caída en sus precios mundiales. Como se observa en la tabla 6, el efecto contractivo del aumento del tipo de cambio real en el modelo estándar es mayor al efecto de la caída de la demanda agregada del modelo estructuralista (-6.1\% vs $-2.2 \%)$.

En la tabla 7 se presentan los resultados en el valor bruto de la producción sectorial de los escenarios simulados. En la segunda y cuarta columna se encuentran los valores obtenidos en el escenario pwagr para los modelos estándar y estructuralista respectivamente. En el primer modelo, el shock inicial deriva en aumentos y disminuciones en los productos sectoriales, dependiendo del grado de intensidad exportadora de las actividades y sus relaciones intersectoriales. El aumento del tipo de cambio real orienta la economía al comercio exterior, las ventas domésticas se reducen $-0.8 \%$, mientras que las exportaciones aumentan $1.9 \%$, con un efecto final expansivo para la mayor parte de las actividades. Los sectores que reducen significativamente su producción son el sector agropecuario, alimentos, refinación de petróleo y construcción. Mientras que los dos primeros caen por la disminución de sus precios internacionales, los combustibles se reducen por la menor demanda interna de los hogares y el sector agropecuario, y la construcción se contrae por la caída en la demanda de inversión ${ }^{13}$.

En el modelo estructuralista, la ausencia de ajustes automáticos conduce a la caída de la producción de todas las actividades, y el efecto negativo final es más profundo que el observado en el modelo estándar. La distribución de esta menor producción entre el mercado doméstico y el externo dependerá de las respectivas elasticidades de transformación. Así, en los casos de mayor elasticidad, como el caso del petróleo y gas, minería, bebidas y tabaco, refinación de petróleo y textil, la caída de los precios domésticos por menor demanda interna posibilitan aumentar las exportaciones, como se observa en la tabla 5. Para el resto de los bienes industriales, el shock produce la caída tanto en las cantidades ofrecidas al mercado interno como externo. Así, en términos agregados las ventas domésticas se reducen $-2.3 \%$, mientras que las exportaciones caen $-2.2 \%$.

\footnotetext{
${ }^{13}$ Los hogares demandan el $38 \%$ del producto de refinación de petróleo. Por su parte, el $88 \%$ del producto construcción se destina a la inversión.
} 
Tabla 7: VBP sectorial, en términos reales (cambio \% respecto de la base)

\begin{tabular}{|c|c|c|c|c|c|c|}
\hline & \multirow[b]{2}{*}{ Base (miles mm $\$$ ) } & \multicolumn{2}{|c|}{ Estándar } & \multicolumn{3}{|c|}{ Estructuralista } \\
\hline & & pwagr & pwagr-ret & pwagr & pwagr-ret & pwagr-dev \\
\hline Agropecuario & 270.963 & $-2.3 \%$ & $-1 \%$ & $-3.9 \%$ & $-2.2 \%$ & $-2.2 \%$ \\
\hline Minería & 48.128 & $0.3 \%$ & $0.3 \%$ & $-0.2 \%$ & $-0.1 \%$ & $0.3 \%$ \\
\hline Hidrocarburos & 88.196 & $0.4 \%$ & $0.4 \%$ & $-0.4 \%$ & $-0.2 \%$ & $0.5 \%$ \\
\hline Electricidad & 116.099 & $-0.3 \%$ & $-0.2 \%$ & $-2.9 \%$ & $-1.7 \%$ & $0.1 \%$ \\
\hline Alimentos & 355.426 & $-4.1 \%$ & $-1.9 \%$ & $-6.7 \%$ & $-4.1 \%$ & $-4.2 \%$ \\
\hline Bebidas y tabaco & 77.557 & $1.5 \%$ & $1.1 \%$ & $-1.3 \%$ & $-0.7 \%$ & $1.9 \%$ \\
\hline Textil & 85.535 & $2.5 \%$ & $1.9 \%$ & $-1.5 \%$ & $-0.8 \%$ & $5.7 \%$ \\
\hline Papel & 50.582 & $0.3 \%$ & $0.4 \%$ & $-2.7 \%$ & $-1.7 \%$ & $1.2 \%$ \\
\hline Refinación petroleo & 99.566 & $-1.1 \%$ & $-0.9 \%$ & $-1.6 \%$ & $-0.9 \%$ & $-0.8 \%$ \\
\hline Quimicos & 148.958 & $1.1 \%$ & $1 \%$ & $-2.7 \%$ & $-1.6 \%$ & $4.1 \%$ \\
\hline Plásticos & 48.687 & $0.8 \%$ & $0.6 \%$ & $-2.7 \%$ & $-1.6 \%$ & $2.9 \%$ \\
\hline Productos metálicos & 105.36 & $1.2 \%$ & $0.2 \%$ & $-1 \%$ & $-0.6 \%$ & $7.6 \%$ \\
\hline Maquinaria & 106.56 & $1.8 \%$ & $0.7 \%$ & $-1.4 \%$ & $-0.8 \%$ & $5.3 \%$ \\
\hline Vehículos & 81.056 & $3.8 \%$ & $3 \%$ & $-1.6 \%$ & $-1 \%$ & $15.2 \%$ \\
\hline Construcción & 299.722 & $-3.9 \%$ & $-5.2 \%$ & $-0.3 \%$ & $-0.2 \%$ & $0.2 \%$ \\
\hline Comercio & 395.77 & $-0.8 \%$ & $-0.7 \%$ & $-3.3 \%$ & $-2 \%$ & $0.8 \%$ \\
\hline Hoteles y restaurantes & 95.906 & $3.8 \%$ & $3 \%$ & $-1.6 \%$ & $-0.8 \%$ & $5.9 \%$ \\
\hline Transporte & 222.065 & $0.4 \%$ & $0.4 \%$ & $-3.1 \%$ & $-1.9 \%$ & $1.3 \%$ \\
\hline Financieros & 140.671 & $-0.6 \%$ & $-0.4 \%$ & $-3.5 \%$ & $-2.2 \%$ & $0.5 \%$ \\
\hline Comunicaciones & 76.57 & $-0.4 \%$ & $-0.2 \%$ & $-4 \%$ & $-2.4 \%$ & $0.7 \%$ \\
\hline Administración pública & 256.529 & $0 \%$ & $0 \%$ & $-0.2 \%$ & $-0.1 \%$ & $0 \%$ \\
\hline Educación & 174.244 & $-0.2 \%$ & $-0.1 \%$ & $-1.3 \%$ & $-0.8 \%$ & $0.2 \%$ \\
\hline Salud & 189.772 & $-0.4 \%$ & $-0.3 \%$ & $-2.9 \%$ & $-1.8 \%$ & $0.2 \%$ \\
\hline Resto & 868.472 & $0.3 \%$ & $0.2 \%$ & $-3.1 \%$ & $-1.9 \%$ & $2 \%$ \\
\hline Total & 4402.394 & $-0.5 \%$ & $-0.4 \%$ & $-2.7 \%$ & $-1.6 \%$ & $1.2 \%$ \\
\hline
\end{tabular}

Fuente: resultados del modelo

\subsection{Reducción de retenciones a las exportaciones}

Una posible respuesta de política ante la caída de los precios internacionales consiste bajar las retenciones a las exportaciones, aumentando el precio de exportación que perciben los productores locales. En el escenario pwagr-ret, se simula la baja en los precios internacionales junto a una reducción del $50 \%$ las retenciones de los productos agropecuarios y de alimentos.

En el modelo estándar, la tabla 7 muestra que la disminución en un $50 \%$ de las retenciones a los productos agropecuarios y de alimentos no modifica significativa- 
mente el nivel de actividad, el empleo y el salario real con respecto al escenario pwagr, pero sí genera cambios en la composición del PBI y el comercio internacional. Los mayores precios percibidos por los productores reducen el impacto negativo inicial observado sobre la exportación agropecuaria y sus manufacturas. Por este motivo, para mantener constante la cuenta corriente el ajuste necesario en el tipo de cambio real es menor, y su incremento pasa del $11.4 \%$ inicial al $9.1 \%$ luego de la política aplicada. Las cantidades exportadas, que luego del shock habían crecido $1.9 \%$, suben hasta el $3.5 \%$, debido a la menor caída en las exportaciones de los sectores agropecuarios y de alimentos, y las importaciones se incrementan 0.7 puntos porcentuales respecto al escenario pwagr. Comparando la segunda y tercer columna de la tabla 7 se observa que la producción de la mayor parte de las actividades cae en relación al shock inicial, y se genera una reasignación de recursos hacia los sectores intensivos en el uso de recursos naturales, en desmedro de los productos industriales. Como se describiera más arriba, en el escenario pwagr-ret el salario real y el nivel de empleo no varían significativamente, pero la reasignación del factor trabajo hacia la producción agropecuaria y alimenticia incrementa la productividad del capital en estos sectores, y en términos agregados el ingreso de capital se incrementa $1 \%$ con respecto al escenario pwagr. El consumo de los hogares se mantiene en terreno negativo luego de la baja en las retenciones, pero gracias al mayor ingreso de capital esta caída es menor a la observada en el escenario pwagr. El consumo de las familias, que había caído $-1.4 \%$ inicialmente, retrocede $-1 \%$ luego de la política, atenuando el efecto negativo en el consumo de los hogares. La menor depreciación del tipo de cambio real con relación al escenario pwagr produce un efecto sustitución que modifica la composición de la canasta de consumo de los hogares, donde adquieren más peso los productos importados en relación a los productos domésticos.

Con respecto al sector público, la combinación de menores precios internacionales con la baja en las retenciones deteriora aún más las cuentas públicas. La recaudación tributaria disminuye y el ahorro del gobierno se reduce un $-97 \%$ respecto al año base. Debido a que el gasto público está fijo en términos reales, el aumento del déficit profundiza la contracción de la inversión, que baja hasta el -8.1\%.

En el modelo estructuralista, la reducción de las retenciones reduce la caída en el nivel de actividad provocado por la baja de los precios internacionales. Al igual que en modelo estándar, pero con magnitudes más elevadas, la economía se ve impulsada por el aumento en las exportaciones del sector agropecuario y de alimentos respecto al escenario pwagr. Estos sectores contratan más mano de obra aumentando el 
empleo y la utilización de capital, y aunque el salario real cae por el incremento de precios internos, se genera un aumento en el ingreso (y en el consumo) real de los hogares respecto al escenario pwagr ${ }^{14}$. La política impositiva logra disminuir el impacto negativo sobre la demanda agregada, y comparando la quinta y sexta columna de la tabla 7 se puede observar que todas las actividades incrementan su producción respecto al shock inicial.

La recaudación tributaria por retenciones disminuye, pero esta situación es compensada con la menor caída en el nivel de actividad, que permite recaudar más que antes de la aplicación de la política impositiva. Sin embargo, la reducción de las retenciones genera aumentos en los precios, aumentando el gasto público nominal. El resultado final es un desahorro del sector público, que pasa del superávit inicial de 25.696 millones, a un déficit luego del shock inicial de -11.892 millones, y un déficit después de la política de -13.560 millones. Finalmente, el incremento de las exportaciones reduce el déficit de cuenta corriente provocado por la caída de los precios mundiales, y la necesidad de financiamiento externo pasa de 54.686 a 49.843 millones.

\subsection{El tipo de cambio como herramienta de política}

En el modelo estructuralista, el tipo de cambio es un instrumento que puede administrarse de acuerdo a los objetivos de política del gobierno. Aunque esto sea así por construcción, la necesidad de financiamiento externo que se produce luego del shock negativo de precios del escenario pwagr es considerable, y alcanza casi los 55.000 millones. En el caso de que el país se encuentre con restricciones en el acceso al crédito internacional el costo de mantener el tipo de cambio fijo se traduce en una pérdida significativa de reservas internacionales o, en última instancia, en una caída en el nivel de actividad. En consecuencia, la administración del tipo de cambio se torna imprescindible para mantener la estabilidad del sistema en el tiempo. En este contexto, en el escenario pwagr-dev se simula un aumento del tipo de cambio nominal del $10 \%$ por encima de los salarios como respuesta de política a los cambios en los precios internacionales ${ }^{15}$.

La devaluación nominal reduce el salario nominal en moneda extranjera, haciendo

\footnotetext{
${ }^{14}$ En términos reales y agregados, los ingresos del trabajo aumentan $0.6 \%$ y los ingresos de capital suben $2.4 \%$ respecto al escenario pwagr.

${ }^{15}$ Para que esta herramienta sea efectiva es necesario que dicho aumento no se traslade totalmente a precios y aumentos salariales, ya que quedaría neutralizado el aumento de competitividad producido por la devaluación nominal.
} 
más competitivas las exportaciones. El aumento del tipo de cambio real del $9.7 \%$ estimula las exportaciones de todos los bienes, y revierte el impacto negativo de los cambios en los precios de las commodities agropecuarias y de alimentos. Las exportaciones totales crecen $3.3 \%$. Los sectores modelados con tecnología Leontief y capacidad ociosa responden al aumento de precios contratando más trabajadores, que se incorporan al proceso productivo con rendimientos constantes. Así, la capacidad de respuesta ante la mejora cambiaria es mayor a aquella que se observaría con funciones de producción con rendimientos decrecientes. Los principales bienes que aumentan sus exportaciones son el sector automotriz $(+18.1 \%)$, el sector textil (18.3\%), productos metálicos (17.3\%), y la extracción de hidrocarburos (13,3\%). La devaluación encarece las importaciones, que a nivel agregado retroceden $-3.2 \%$. Cae la demanda importada de todos los productos menos los agropecuarios y alimentos, donde persiste la baja de precios internacionales. Se produce un efecto sustitución de consumo importado por doméstico en los hogares y la inversión ${ }^{16}$, estimulando la producción nacional.

Como consecuencia, se revierte la baja del PBI, que se incrementa en $0.9 \%$, y la mayor demanda presiona a la suba de los precios internos, que erosiona el salario real. El desempleo baja al 6.4\%, y se producen reasignaciones del factor trabajo entre actividades. El ingreso real total de los hogares retrocede - $0,8 \%$, bajando su consumo real en $-0.1 \%$. El efecto expansivo de la devaluación aumenta la recaudación del gobierno con respecto al shock inicial, a la vez que el aumento de precios producido por la devaluación impacta en el gasto nominal del gobierno. El resultado neto es que el ahorro del sector público logra mantenerse sin cambios significativos con respecto al año base. De esta manera, el aumento de las exportaciones y el equilibrio del sector público reducen la necesidad de financiamiento externo que había provocado la baja de precios internacionales. El ahorro del resto del mundo, que luego del shock alcanzó los 54.686 millones, pasa a 8.182 millones luego de la política cambiaria.

\section{Conclusiones}

La dinámica de la economía argentina ha estado siempre condicionada por su inserción en el comercio internacional. A partir de los años 50, se consolidó una estructura industrial con alto grado de dependencia de la importación de bienes de capital e in-

\footnotetext{
${ }^{16}$ Las cantidades importadas demandadas de las familias bajan un $10 \%$, y la inversión también sustituye bienes importados por domésticos, bajando sus importaciones un $7 \%$.
} 
termedios. En este marco, la actividad agropecuaria y sus manufacturas fueron clave para obtener las divisas necesarias para el funcionamiento de la industria. El proceso económico de la última década no logró modificar significativamente el entramado industrial histórico, y a partir del año 2012 la restricción externa volvió a presentarse como un serio limitante para el crecimiento. A esta situación estructural interna se le sumaron los cambios que en 2014 se registraron en los precios internacionales, donde la caída de los precios de las commodities agropecuarias repercutieron en la balanza de pagos, agravando la necesidad de financiamiento externo.

En el presente trabajo se construyeron dos modelos de equilibrio general computable, que fueron calibrados para la economía argentina del año 2012 a los fines de evaluar los efectos económicos de los cambios en los precios internacionales de las commodities. Posteriormente se analizaron algunas alternativas de política para atenuar el efecto contractivo en la demanda agregada y la necesidad de financiamiento externo. Se evaluó en primera instancia un modelo estándar y posteriormente se replicaron los shocks para un modelo estructuralista, con el objetivo de comparar los resultados que se obtienen al utilizar distintos supuestos, tanto a nivel de comportamiento micro como a nivel macroeconómico.

A nivel micro, las principales diferencias están vinculadas a las tecnologías de producción, formación de precios y el grado de utilización del capital. En el modelo estructuralista la combinación de desempleo con capacidad ociosa permite responder a los cambios en la demanda agregada incorporando o reduciendo la cantidad de trabajadores con rendimientos constantes. El poder de mercado fija el margen de beneficios de las firmas, y el ajuste de cantidades ante cambios de demanda es más fuerte que el ajuste de precios. Combinando estos factores, las variaciones en la demanda producen reacciones de cantidades superiores a las obtenidas con el modelo estándar. Por su parte, el modelo estándar también supone desempleo en el mercado laboral, pero los factores se incorporan a la producción con rendimientos decrecientes. Las firmas no tienen poder de mercado, y las modificaciones en la demanda producen ajustes de precios superiores a los observados en el caso estructuralista.

A nivel macro, se destacan las diferencias que el ajuste del sector externo y el equilibrio del ahorro y la inversión generan en los resultados de las simulaciones. En el cierre estándar el tipo de cambio real es la variable que ajusta para equilibrar el sector externo, y la devaluación del tipo de cambio real conduce al aumento de las exportaciones y la disminución de las importaciones. En el caso estructuralista, el tipo de cambio real definido en función del salario está fijo, y el ajuste se produce 
mediante cambios en el ahorro del resto del mundo. Esta especificación brinda una medida de la necesidad de financiamiento externo ante shocks exógenos.

Analizando los resultados de las simulaciones, en el modelo estándar una disminución del $25 \%$ del precio de las commodities agropecuarias produce un aumento del tipo de cambio real del $11.3 \%$. La producción, estimulada por el aumento de competitividad cambiaria, se orienta al mercado externo, disminuyendo el consumo de los hogares y la inversión. El resultado sobre el PBI es levemente contractivo, cayendo $-0.5 \%$, y tiene consecuencias negativas sobre la acumulación de capital, que condiciona el crecimiento futuro de la economía.

En el modelo estructuralista, el shock negativo de precios contrae la demanda agregada, reduciendo el nivel de actividad un $-2.7 \%$. La caída en los ingresos de los hogares y el gobierno potencia el efecto inicial, y el financiamiento de la inversión se produce mediante un aumento del ahorro del resto del mundo. La necesidad de financiamiento externo asciende a los 54.638 millones, que en la práctica debe cubrirse con endeudamiento externo o diminución de reservas internacionales. La baja en las retenciones como respuesta de política tiene efectos diferentes entre modelos. En el modelo estándar no produce cambios significativos en el PBI, pero genera una mayor especialización de la economía hacia sectores intensivos en recursos naturales, y disminuye la inversión por el aumento del déficit público. En el caso estructuralista, la baja de las retenciones amortigua la caída inicial del PBI, y la menor percepción del gobierno por retenciones se compensa con la mayor recaudación por aumento en el nivel de actividad. Sin embargo, la necesidad de financiamiento externo continua siendo elevada, y luego de la política tributaria el aumento del ahorro del resto del mundo asciende a los 49.843 millones.

Utilizando el modelo estructuralista se simuló una devaluación del tipo de cambio nominal del $10 \%$, que permitió contrarrestar el efecto contractivo de la demanda agregada. Luego de la devaluación, el PBI se incrementó $0.9 \%$, impulsado por el aumento de las exportaciones y la sustitución de consumo importado por doméstico. El cambio en el ahorro del resto del mundo se redujo a 8.182 millones, cifra significativamente menor a la resultante del shock inicial de precios. Cabe destacar que utilizar el tipo de cambio nominal como herramienta de política no está exenta de conflictos distributivos. Para que la administración cambiaria sea efectiva este aumento no debe trasladarse a precios y salarios.

Finalmente, la caída en los precios internacionales de las commodities utilizando diferentes marcos conceptuales conduce a resultados sensiblemente diferentes. El 
modelo estándar genera ajustes automáticos que pueden no verificarse en el corto plazo, ya que el tipo de cambio en Argentina es comúnmente utilizado como variable de política por las autoridades económicas. Sin embargo, si no se cuenta con acceso al crédito, la necesidad de financiamiento externo puede llevar a las reservas internacionales a niveles críticos. De esta manera, las recomendaciones de política resultantes de estos modelos de equilibrio general computable dependerán tanto de los supuestos utilizados como del marco institucional en el que son desarrollados. Considerando las características de la economía argentina, el modelo estructuralista proporciona herramientas que permiten evaluar los efectos de corto plazo, en donde los resultados obtenidos dependerán del contexto institucional en que se encuentra inmerso. Aun así, la exogeneidad de variables clave para la economía, como el tipo de cambio nominal, los salarios, la inversión, los ingresos y los gastos del sector público, permiten controlar y cuantificar los resultados de las decisiones de política económica utilizadas para administrar la restricción externa y darle sustentabilidad de largo plazo al sistema económico. 


\section{Referencias}

[1] Basualdo, Eduardo M. (2006). Estudios de historia económica argentina: desde mediados del siglo XX a la actualidad. FLACSO.

[2] CEPAL (2014), Balance Económico Actualizado de América Latina y el Caribe 2013, Chile.

[3] Chisari O. O., C. A. Romero, G. Ferro, R. Theller, M. Cicowiez, J. Ferraro, M.González, A. Blanco y J. Maquieyra (2009). Un Modelo de Equilibrio GeneralComputable para la Argentina. PNUD Argentina.

[4] Chisari, Omar, Mastronardi, Leonardo, y Romereo, Carlos (2014). Commodities prices and critical parameters for macroeconomic performance: a CGE analysis for Argentina, Brazil and Chile. XLIX Reunión Anual de la Asociación Argentina de Economía Política, Rosario.

[5] Cicowiez, Martín y Mercado, P. Rubén (2009). Modelos y Escenarios Multisectoriales. En B. Kosacoff y P. R. Mercado (eds.). La Argentina ante la Nueva Internacionalización de la Producción: Crisis y Oportunidades. CEPAL y PNUD.

[6] Cicowiez, Martín (2011), Un Modelo de Equilibrio General Computado para la Evaluación de Políticas Económicas en Argentina: Construcción y Aplicaciones. Tesis de Doctorado, UNLP.

[7] Dervis, Kemal, Jaime de Melo and Sherman Robinson (1982). General EquilibriumModels for Development Policy. Cambridge: Cambridge University Press.

[8] De Melo, Jaime, and Robinson, Sherman (1989). Product differentiation and the treatment of foreign trade in computable general equilibrium models of small economies, Journal of International Economics 27 (1989) 47-67. North-Holland

[9] Diamand, M. (1972). La estructura productiva desequilibrada argentina y el tipo de cambio, Desarrollo Económico, Vol. 12, No 45, pp. 45-47.

[10] Di Gresia, Luciano (2009). Impacto Federal de Reformas Tributarias. Una Aproximación de Equilibrio General Computado. Anales Reunión Anual Asociación Argentina de Economía Política.

[11] Harberger, Arnold C. (1962). The Incidence of the Corporation Income Tax. Journal of Political Economy 70 (3): 215-240.

[12] Harris, Richard (1984), "Applied General Equilibrium Analysis of Small Open Economies with Scale Economies amd Imperfect Competition", American Economic Review, vol. 74(5), pp. 1016-1032.

[13] Johansen, Leif (1960). A Multi-Sectoral Study of Economic Growth. Amsterdam: North-Holland. 
[14] Lofgren, Hans, Rebecca Lee Harris and Sherman Robinson (2002). A Standard Computable General Equilibrium (CGE) Model in GAMS. International Food Policy Research Institute (IFPRI) Microcomputers in Policy Research 5.

[15] Rapoport, Mario (2008). Historia económica, política y social de la Argentina, 18802003, Editorial Emecé, Buenos Aires.

[16] Robinson, Sherman (1989). Multisectoral Models. En H. Chenery y T. N. Srinivasan (eds.). Handbook of Development Economics (Vol. 2).Amsterdam: North Holland.

[17] Robinson, Sherman (2006). Macro Models and Multipliers: Leontief, Stone, Keynes, and CGE Models. deJanvry, Alain y Kanbur, Ravi (eds.). Poverty, Inequality and Development: Essays in Honor of Erik Thorbecke. New York: Springer Science.

[18] Serino, Leandro Antonio (2009). Productive Diversification in Natural Resource Abundant Countries. Shaker Publishing 2009.

[19] Taylor, Lance; Bacha, Edmar L.; Cardoso , Eliana; Lysy, Frank (1980). Models of Growth and Distribution for BrazilWord Bank - Oxford University Press.

[20] Taylor, Lance (1990). Socially Relevant Policy Analysis. Structuralist Computable General Equilibrium Models for the Developing. The MIT Press.

[21] Taylor, L. (2004) Reconstructing Macroeconomics: Structuralist Proposals and Critiques of the Mainstream, Cambridge, Massachusetts: Harvard University Press.

[22] Taylor. Lance, y Von Arnim, Rudiger (2006), Modelling the Impact of Trade Liberalisation. A Critique of Computable General Equilibrium Models

[23] Von Arnim, Rudiger (2009). Recession and rebalancing: How the housing and credit crises will impact US real activity, University of Denver, Department of Economics, 2000 E. Asbury, Denver, CO 80208, United States.

[24] Von Arnim, Rudiger, y Rada, Codrina (2011). Labour Productivity and Energy Use in a Three-SectorModel: An Application to Egypt, Development and Change, Institute of Social Studies. 


\section{A. ANEXO 1: Los modelos}

\section{Actividades con mark up}

$$
\begin{gathered}
P A_{a}=\left(1+T A O_{a}\right)\left(1+t a_{a}\right) C V_{a} \\
C V_{a}=\sum_{l a b} W F_{l a b} W F D I S T_{l a b, a}+\sum_{c} i c a_{c, a} P Q D_{c, a} \\
b_{l a b, a}=\frac{Q F_{l a b, a}}{Q A_{a}} \\
U_{c a p, a}=\frac{Q A_{a}}{Q F_{c a p, a}} \\
W F_{f} W F D I S T_{f, a}=P A_{a} \frac{T A O_{a} U_{c a p, a}}{\left(1+T A O_{a}\right)\left(1+t a_{a}\right)}
\end{gathered}
$$

\section{Actividades competitivas}

$$
\begin{gathered}
Q V A_{a}=\pi_{a} \sum_{c}\left[\delta_{f, a}^{v a} Q F_{f, a}^{-\rho v a_{a}}\right]^{-\frac{1}{\rho v a_{a}}} \\
\frac{Q F_{f, a}}{Q V A_{a}}=\left(\frac{P V A_{a}}{W F_{f} W F D I S T_{f, a}}\right)^{\sigma v a_{a}}\left(\delta_{f, a}^{v a}\right)^{\sigma v a_{a}} \pi_{a}^{\left(\sigma v a_{a}-1\right)} \\
P A_{a}\left(1-t a_{a}\right) Q A_{a}=P V A_{a} Q V A_{a}+P I N T A_{a} Q I N T A_{a} \\
Q V A_{a}=i v a_{a} Q A_{a}
\end{gathered}
$$

\section{Precios y valor agregado}

$$
\begin{aligned}
P M_{c, a c} & =\left(1+t m_{c, a c}\right) E X \operatorname{Rpwm}_{c} \\
P E_{c} & =\left(1-t e_{c}\right) E X \text { Rpwe }_{c}
\end{aligned}
$$




$$
\begin{aligned}
& Q X_{a}=\sum_{c} \theta_{a, c} Q A_{c} \\
& P A_{a}=\sum_{c} \theta_{a, c} P X_{c}
\end{aligned}
$$

\section{Índice de precios y tipo de cambio real}

$$
\begin{gathered}
\sum_{c} c w t s_{c, h} P Q D_{c, a c}=C P I \\
\sum_{c} d w t s_{c} P D_{c}=D P I \\
R E X R=\frac{E X R}{D P I}
\end{gathered}
$$

\section{Bien Compuesto Consumo}

$$
\begin{gathered}
Q Q_{c, a c}=a q_{c, a c}\left[\delta q_{c, a c}^{M} Q M_{c, a c}^{-\rho q_{c, a c}}+\delta q_{c, a c}^{D} Q D_{c, a c}^{-\rho q_{c, a c}}\right]^{-\frac{1}{\rho q_{c, a c}}} \\
\frac{Q M_{c, a c}}{Q D_{c, a c}}=\left(\frac{P D_{c}}{P M_{c, a c}} \frac{\delta q_{c, a c}^{M}}{\delta q_{c, a c}^{D}}\right)^{\frac{1}{\left(1+\rho q_{c}, a c\right)}} \\
P Q S_{c, a c} Q Q_{c, a c}=P M_{c, a c} Q M_{c, a c}+P D_{c, a c} Q D_{c, a c} \\
Q Q_{c, a c}=Q D_{c, a c}+Q M_{c, a c} \\
P Q D_{c, a c}=P Q S_{c, a c}\left(1+t q_{c, a c}\right)
\end{gathered}
$$

Bien Compuesto Producción

$$
Q X_{c}=a t_{c}\left[\delta t_{c}^{E} Q E_{c}^{\rho t_{c}}+\delta t_{c}^{D} Q D S_{c}^{\rho t_{c}}\right]^{\frac{1}{\rho t_{c}}}
$$




$$
\begin{gathered}
\frac{Q E_{c}}{Q D S_{c}}=\left(\frac{P E_{c}}{P D_{c}} \frac{\delta t_{c}^{D}}{\delta t_{c}^{E}}\right)^{\frac{1}{\left(\rho t_{c}-1\right)}} \\
P X_{c} Q X_{c}=P D S_{c} Q D S_{c}+P E_{c} Q E_{c} \\
Q X_{c}=Q D S_{c}+Q E_{c}
\end{gathered}
$$

Variación de existencias

$$
\begin{gathered}
Q Q_{c, d s t k}=Q M_{c, d s t k}+Q D_{c, d s t k} \\
Q D_{c, d s t k}=q d s t d_{c} \\
Q M_{c, d s t k}=q d s t m_{c}
\end{gathered}
$$

Demanda inversión y bienes intermedios

$$
\begin{gathered}
i c a_{c, a}=\frac{Q I N T_{c, a}}{Q I N T A_{a}} \\
i n t a_{a}=\frac{Q I N T A_{a}}{Q A_{a}} \\
P I N T A_{a}=\sum_{c} i c a_{c, s} P Q D_{c, a} \\
Q I N V_{c}=\text { qinvbar }_{c} I A D J
\end{gathered}
$$

Instituciones domésticas distintas al gobierno

$$
\begin{gathered}
Y F_{f}=\sum_{a} W F_{f} Q F_{f, a} W F D I S T_{f, a}+t r n s f r_{f, r o w} E X R \\
Y I F_{\text {ins }, f}=\operatorname{shif} f_{\text {ins }, f}\left(1+t f_{f}\right) Y F_{f}
\end{gathered}
$$




$$
\begin{gathered}
Y I_{\text {insdng }}=\sum_{f} Y I F_{\text {insdng,f }}+t r n s f r_{\text {insdng,gov }} C P I \\
+ \text { trnsf } r_{\text {insdng, row }} E X R+\sum_{f} T R I I_{\text {insdng,insdng }} \\
T R I I_{\text {ins,insdng }}=\text { shii } i_{\text {ins }, \text { insdng }}\left(1-M P S_{i} n s d n g\right)\left(1-t y_{\text {insdng }}\right) Y I_{\text {insdng }} \\
M P S_{\text {insdng }}=m p s b a r_{\text {insdng }} M P S A J \\
E H_{h}=\left(1-\sum_{i n s} s h i i_{i n s, h}\right)\left(1-M P S_{h}\right)\left(1-t y_{h}\right) Y I_{h} \\
Q H_{c, h}=\left(\alpha_{c, h} E H_{h}\right) / P Q D_{c, h}
\end{gathered}
$$

\section{Gobierno}

$$
\begin{array}{r}
T R E V=\sum_{c} t q_{c, a c} Q Q_{c, a c} P Q S_{c, a c} \\
+\sum_{\text {insdng }} t y_{\text {insdng }} Y I_{\text {insdng }}+\sum_{c} t_{c, a c} Q M_{c, a c} p w m_{c} E X R \\
+\sum_{c} t e_{c} Q E_{c} p w e_{c} E X R \\
+\sum_{a} T A O_{a} t a_{a} C V_{a}+\sum_{a} P A_{a} t a_{a} Q A_{a}+\sum_{f} t f_{f} Y F_{f} \\
+\sum_{i n s d n g} T R I I_{\text {gov }, \text { insdng }}+\sum_{f} Y I F_{\text {gov }, f} \\
E G=\sum_{c} Q G_{c} P Q D_{c, g o v} \\
+\sum_{i} t r n s f r_{\text {insdng,gov }} C P I+E X R t r n s f r_{\text {row }, g o v}
\end{array}
$$




$$
Q G_{c}=q g b a r_{c} * G A D J
$$

$$
G S A V=Y G-E G
$$

\section{Ecuaciones de balance}

Equilibrio mercado de factores

$$
Q F S_{f}=\sum_{a} Q F_{f, a}
$$

Equilibrio mercado de bienes

$$
\begin{gathered}
Q I N T_{c, a}=Q Q_{c, a} \\
Q H_{c, h}=Q Q_{c, a} \\
Q I N V_{c}=Q Q_{c, s i} \\
Q G_{c}=Q Q_{c, g o v} \\
Q D S T_{c}=Q Q_{c, d s t k} \\
Q D S_{c}=\sum_{a c} Q D_{c, a c}
\end{gathered}
$$

\section{Equilibrio ahorro-inversión}

$$
\begin{aligned}
& \sum_{\text {insdng }}\left(1-t y_{\text {insdng }}\right) M P S_{\text {insdng }} Y H_{\text {insdng }}+G S A V+E X R F S A V \\
& \left.\quad=\sum_{c} Q I N V_{c} P Q D_{c, s i}+\sum_{c} Q D S T_{c} P Q D_{(} c, s i\right)+W A L R A S
\end{aligned}
$$




\section{Cuenta corriente}

$$
\begin{aligned}
& \sum_{c} p w e_{c} Q E_{c}+\sum_{\text {insd }} t r n s f r_{\text {insd,row }}+\sum_{f} \text { trnsfr } r_{f, \text { row }} \\
& +F S A V=\sum_{c} \sum_{a c} Q M_{c, a c} p w m_{c, a c}+\text { trnsfr } r_{\text {row }, \text { gov }} \\
& +\sum_{f} \frac{Y I F_{\text {row }, f}}{E X R}+\sum_{\text {insdng }} \frac{T R I I_{\text {row }, \text { insdng }}}{E X R}
\end{aligned}
$$

\section{Mercado laboral del modelo estándar}

$$
\begin{gathered}
W F R E A L_{l a b}=\frac{W F_{l a b}}{C P I} \\
\frac{W F R E A L_{l a b}}{W F R E A L 0_{l a b}}-1=- \text { phillips }_{l a b}\left(\frac{U E R A T_{l a b}}{U E R A T 0_{l a b}}-1\right) \\
Q F S_{l a b}\left(1-U E R A T_{l a b}\right)=\sum_{a} Q F_{l a b, a}
\end{gathered}
$$




\section{Conjuntos}

$\begin{array}{cl}a c & \text { todas las cuentas de la sam } \\ a & \text { actividades } \\ \text { amk } \in a & \text { actividades mark up } \\ \text { anmk } \in a & \text { actividades no mark up } \\ c & \text { productos } \\ f & \text { factores } \\ f_{\text {lab }} & \text { factor trabajo } \\ f_{\text {cap }} & \text { factor capital } \\ \text { insd } & \text { instituciones domesticas } \\ \text { insdng } & \text { instituciones domesticas no gobierno } \\ \text { gov } & \text { gobierno } \\ \text { row } & \text { resto del mundo } \\ h & \text { hogares } \\ \text { dstk } & \text { variación de existencias } \\ \text { si } & \text { ahorro-inversión }\end{array}$

\section{Variables}

$P A_{a} \quad$ Precio de la actividad a

$\mathrm{TAO}_{a} \quad$ Tasa mark up de la actividad a

$W F_{f} \quad$ precio del factor $\mathrm{f}$

$W F D I S T_{f, a}$ factor distorsión precio del factor $\mathrm{f}$ de la actividad a

$P E_{c} \quad$ precio del bien c exportación moneda domestica

$P D_{c} \quad$ precio del bien c domestico

$P M_{c, a c} \quad$ precio del bien c importación moneda doméstica agente ac

$P X_{c} \quad$ precio del productor bien c

EXR tipo de cambio (moneda doméstica por unidad de moneda resto mundo) 
$P V A_{a} \quad$ precio del valor agregado actividad a

$C V_{a} \quad$ Costo variable

$P Q D_{c, a c} \quad$ precio demanda del bien compuesto c agente ac

$P Q S_{c, a c} \quad$ precio oferta del bien compuesto c agente ac

$Q A_{a} \quad$ nivel de actividad a

$Q F_{f, a} \quad$ demanda del factor $\mathrm{f}$ de actividad a

$Q F S_{f, a} \quad$ oferta total del factor $\mathrm{f}$

QINT $T_{c, a}$ consumo intermedio del bien c de la actividad a

$Q D S_{c} \quad$ ventas del bien c domestico

$Q D_{c, a c} \quad$ compras del bien c domestico del agente ac

$Q E_{c} \quad$ exportaciones del bien c

$Q M_{c, a c} \quad$ importaciones del bien c agente ac

$Q Q_{c, a c} \quad$ demanda doméstica del bien compuesto c del agente ac

$Q X_{c} \quad$ oferta domestica del bien compuesto c

$Q H_{c, h} \quad$ consumo del bien c hogar h

$\mathrm{EH}_{h} \quad$ gasto de consumo hogar h

$U_{\text {cap }, a} \quad$ capacidad de utilización

$Q I N V_{c} \quad$ demanda para inversión del bien c

$M P S_{i} n s \quad$ propensión marginal a ahorrar institución ins

$I A D J \quad$ factor de ajuste de la inversión

$M P S A D J$ factor de ajuste de la propensión marginal ahorrar

$Y G \quad$ ingreso del gobierno

$E G \quad$ gasto del gobierno

FSAV ahorro del resto mundo (moneda resto mundo)

$Q G_{c} \quad$ consumo del gobierno bien c

$G A D J \quad$ factor de ajuste del consumo gobierno

$G S A V \quad$ ahorro del gobierno

QINTA $A_{a}$ agregado insumos intermedios de la actividad a

$Q V A_{a} \quad$ valor agregado de la actividad a

PINT $A_{a}$ precio agregado de insumos intermedios de la actividad a $Y F_{h, f} \quad$ ingreso del factor $\mathrm{f}$ 


$$
\begin{array}{cl}
Y I F_{\text {ins }, f} & \text { ingreso institución ins del factor } \mathrm{f} \\
Y I_{i} n s & \text { ingreso de la institución insdng } \\
T R I I_{\text {ins,ins }} & \text { transferencia desde insdng hacia ins } \\
T R E V & \text { recaudación tributaria } \\
W A L R A S & \text { variable para cumplir ley de Walras } \\
R E X R & \text { tipo de cambio real } \\
Q D S T_{c} & \text { variación existencias del bien c } \\
C P I & \text { Indice de precios al consumidor } \\
D P I & \text { Indice de precios domésticos del productor }
\end{array}
$$

\section{Parámetros}

$\begin{array}{cl}t a_{a} & \text { tasa de impuesto a la producción actividad a } \\ t q_{c, a c} & \text { tasa de impuesto a ventas bien c agente ac } \\ t y_{i} n s & \text { tasa de impuesto a ingreso institución ins } \\ t e_{c} & \text { tasa de impuesto a exportaciones bien c } \\ t m_{c, a c} & \text { tasa de arancel a bien c agente ac } \\ t f_{f} & \text { tasa de impuesto a ingreso factor } \mathrm{f} \\ \text { shif }_{\text {ins,f }} & \text { participación de la institución ins en ingreso factor } \mathrm{f} \\ q i n v b a r_{c} & \text { demanda para inversión del bien c inicial } \\ m p s b a r_{i n s d n g} & \text { propensión marginal ahorrar hogar h inicial } \\ q g b a r_{c} & \text { consumo gobierno bien c inicial } \\ t r n s f r_{a c, i n s} & \text { transferencia desde institución insp a cuenta ac } \\ s h i i_{i n s, i n s} & \text { participación de transferencias entre instituciones en ingreso } \\ \text { pwe }_{c} & \text { de la institución ins } \\ \text { pwm }_{c} & \text { precio de exportación del bien c (moneda resto mundo) } \\ q d s t d_{c} & \text { varecio de importación del bien c (moneda resto mundo) } \\ q d s t m_{c} & \text { variación de existencias importadas del bien c } \\ \delta_{f, a}^{v a} & \text { participación del factor f en valor agregado de la actividad a } \\ \pi_{a} & \text { parámetro de escala en valor agregado de la actividad a }\end{array}$


$\sigma v a_{a}$ elasticidad sustitución en valor agregado de la actividad a

$\rho v a_{a} \quad$ exponente función valor agregado de la actividad a

$\theta_{a, c} \quad$ producción de bien c por unidad de actividad a

$i c a_{c, a} \quad$ consumo intermedio de bien c por unidad insumos intermedios actividad a

$i v a_{a} \quad$ valor agregado por unidad de actividad a

inta $_{a}$ insumos intermedios por unidad de actividad a

$\alpha_{c, h} \quad$ participación del bien c en el consumo del hogar $\mathrm{h}$

$\delta q_{c, a c}^{M} \quad$ participación de bien importado c en armington q agente ac

$\delta q_{c, a c}^{D} \quad$ participación de bien domestico c en armington q agente ac

$a q_{c, a c} \quad$ parámetro de escala en armington q agente ac

$\sigma q_{c, a c} \quad$ elasticidad sustitución armington q agente ac

$\rho q_{c, a c} \quad$ exponente de la función armington q agente ac

$\delta t_{c}^{E} \quad$ participación de las exportaciones de bien c en cet $\mathrm{x}$

$\delta t_{c}^{D} \quad$ participación del bien domestico c en cet $\mathrm{x}$

$a t_{c} \quad$ parámetro de escala en cet $\mathrm{x}$

$\sigma t_{c} \quad$ elasticidad de transformación cet $\mathrm{x}$

$\rho t_{c} \quad$ exponente de lafunción cet $\mathrm{x}$

$c w t s_{c, h}$ ponderación del bien c en la canasta de consumo del hogar $\mathrm{h}$

$d w t s_{c} \quad$ ponderación del bien c en índice de precios al productor

$b_{l, a} \quad$ coeficiente de demanda laboral Leontief 


\section{B. ANEXO 2: Especificaciones y elasticidades}

Tabla 8: Especificaciones del modelo estructuralista

\begin{tabular}{|c|c|c|c|}
\hline Código & Actividad & Código & Actividad \\
\hline Administración Pública & \multirow{10}{*}{$\begin{array}{l}\text { Producción } \\
\text { eficiente, } \\
\text { firmas } \\
\text { competitivas }\end{array}$} & Productos Metálicos & \multirow{16}{*}{$\begin{array}{l}\text { Producción } \\
\text { con } \\
\text { capacidad } \\
\text { ociosa, } \\
\text { firmas con } \\
\text { poder de } \\
\text { mercado }\end{array}$} \\
\hline Agropecuario & & Maquinaria & \\
\hline Forestal & & Vehículos & \\
\hline Pesca & & Maquinaria & \\
\hline Minería & & Vehículos & \\
\hline Petróleo y gas & & Otros manufacturados & \\
\hline Electricidad & & Construcción & \\
\hline Alimentos & & Comercio & \\
\hline Bebidas y Tabaco & & Hoteles y restaurantes & \\
\hline Madera & & Transporte & \\
\hline Textil & \multirow{6}{*}{$\begin{array}{l}\text { Producción } \\
\text { con } \\
\text { capacidad } \\
\text { ociosa, } \\
\text { firmas con } \\
\text { poder de } \\
\text { mercado }\end{array}$} & Agua & \\
\hline Refinación dePetróleo & & Financieros & \\
\hline Químicos & & Otros servicios & \\
\hline Plásticos & & Comunicaciones & \\
\hline Minerales no Metálicos & & Educación & \\
\hline Metálicos Básicos & & Salud & \\
\hline
\end{tabular}

Fuente: elaboración propia en base a INDEC y Serino (2009) 
Tabla 9: Elasticidades de sustitución sectoriales, función CET y de producción CES

\begin{tabular}{lcc}
\hline & sigmat & sigmaVA \\
\hline Agropecuario & 0.415 & 0.24 \\
Forestal & 0.832 & 0.24 \\
Pesca & 0.832 & 0.24 \\
Minería & 1.9 & 0.2 \\
petróleo y gas & 3.8 & 0.2 \\
Electricidad & 2.8 & 0.668 \\
Alimentos & 1.233 & 0.668 \\
bebidas y tabaco & 1.233 & 0.72 \\
Textil & 1.194 & 1.05 \\
Madera & 0.832 & 1.05 \\
Papel & 0.832 & 0.229 \\
refinación de petróleo & 0.928 & 0.229 \\
Químicos & 0.861 & 0.805 \\
Pláticos & 1.042 & 0.694 \\
Minerales no metálicos & 1.048 & 0.694 \\
Otras manufacturas & 0.653 & 1.05 \\
Metálicos básicos & 0.653 & 0.878 \\
Productos metálicos & 1.056 & 0.342 \\
Maquinaria & 0.701 & 0.714 \\
Vehículos & 0.383 & 0.896 \\
Construcción & 1.9 & 0.896 \\
Comercio & 1.9 & 0.896 \\
Hoteles y restaurantes & 1.9 & 1.05 \\
Transporte & 1.9 & 0.866 \\
Gas & 2.8 & 0.866 \\
Agua & 2.8 & 1.05 \\
Financieros & 2.8 & 0.441 \\
Otros servicios & 1.9 & 1.05 \\
Comunicaciones & 0.775 \\
Educinistración pública & 1.05 \\
\hline
\end{tabular}

Fuente: Cicowiez (2011) 